TRANSACTIONS OF THE

AMERICAN MATHEMATICAL SOCIETY

Volume 350, Number 8, August 1998, Pages 3269-3296

S 0002-9947(98)01971-0

\title{
A FAMILY OF QUANTUM PROJECTIVE SPACES AND RELATED $q$-HYPERGEOMETRIC ORTHOGONAL POLYNOMIALS
}

\author{
MATHIJS S. DIJKHUIZEN AND MASATOSHI NOUMI
}

\begin{abstract}
A one-parameter family of two-sided coideals in $\mathcal{U}_{q}(\mathfrak{g l}(n))$ is defined and the corresponding algebras of infinitesimally right invariant functions on the quantum unitary group $U_{q}(n)$ are studied. The Plancherel decomposition of these algebras with respect to the natural transitive $U_{q}(n)$-action is shown to be the same as in the case of a complex projective space. By computing the radial part of a suitable Casimir operator, we identify the zonal spherical functions (i.e. infinitesimally bi-invariant matrix coefficients of finite-dimensional irreducible representations) as Askey-Wilson polynomials containing two continuous and one discrete parameter. In certain limit cases, the zonal spherical functions are expressed as big and little $q$-Jacobi polynomials depending on one discrete parameter.
\end{abstract}

\section{INTRODUCTION}

In this paper, we study a family of two-sided coideals $\mathfrak{k}^{(c, d)}(c, d$ non-negative real numbers) in the quantized universal enveloping algebra $\mathcal{U}_{q}(\mathfrak{g l}(n))$. The coideals $\mathfrak{k}^{(c, d)}$ can be viewed as a $q$-analogue of the Lie subalgebra $\mathfrak{g l}(n-1) \oplus \mathfrak{g l}(1) \subset \mathfrak{g l}(n)$. By considering the algebra of functions on the quantum unitary group $U_{q}(n)$ that are "infinitesimally" invariant with respect to the coideal $\mathfrak{k}^{(c, d)}$, we obtain a family of quantum projective spaces $\mathbb{C P}_{q}^{n-1}(c, d)$ endowed with a natural transitive action of the quantum unitary group $U_{q}(n)$. These quantum $U_{q}(n)$-spaces were studied for the first time by Vaksman and Korogodsky [KV], who defined them in a "global" way by means of a $q$-analogue of the classical Hopf fibration $S^{2 n-1} \rightarrow \mathbb{C P}^{n-1}$. We also analyse the zonal spherical functions (infinitesimally left $\mathfrak{k}^{(c, d)}$-invariant and right $\mathfrak{k}^{\left(c^{\prime}, d^{\prime}\right)}$-invariant matrix coefficients) corresponding to finite-dimensional irreducible representations of $U_{q}(n)$. They are expressed in terms of a family of Askey-Wilson polynomials containing two continuous and one discrete parameter. We obtain this result by showing that the zonal spherical functions are eigenfunctions of a certain second-order $q$-difference operator which arises as the radial part of a suitable Casimir operator.

Received by the editors April 28, 1996 and, in revised form, October 1, 1996.

1991 Mathematics Subject Classification. Primary 33D80, 81R50, 17B37, 33D45.

Key words and phrases. Quantum unitary group, quantum projective space, two-sided coideal, zonal spherical function, Casimir operator, radial part, second-order $q$-difference operator, AskeyWilson polynomials, big and little $q$-Jacobi polynomials.

The first author acknowledges financial support by the Japan Society for the Promotion of Science (JSPS) and the Netherlands Organization for Scientific Research (NWO).

(C)1998 American Mathematical Society 
The method of constructing quantum homogeneous spaces by using the notion of "infinitesimal" invariance with respect to the natural action of the quantized universal enveloping algebra was introduced by Koornwinder [K2], [K3] in the case of the quantum $S U(2)$ group. He actually worked with so-called twisted primitive elements in the quantized universal enveloping algebra $\mathcal{U}_{q}(\mathfrak{s l}(2))$. For other applications of this idea to the $S U_{q}(2)$ case we refer to [NM2], [Kk], [DK1]. By extending the notion of twisted primitive element to the more general one of two-sided coideal, it was shown in subsequent papers (cf. [N], [S1], [NS1]; see also [DK1]) that the infinitesimal method could also be fruitfully applied in the case of higher-dimensional quantum groups. It not only provides one with a more practical way to construct examples of quantum homogeneous spaces (finding a suitable two-sided coideal is easier than constructing the algebra of functions on a quantum homogeneous space by means of generators and relations), but it also allows one to study infinitesimally bi-invariant functions or (zonal) spherical functions, something which can be done to only a very limited extent if the quantum homogeneous space has been defined in a "global" way.

The results of this paper constitute a simultaneous generalization of results in various other papers. First of all, the case $c=1, d=0$ ( $n$ arbitrary) was dealt with by Mimachi, Yamada and the second author [NYM] (see also Vaksman and Sořbel'man [VS2]). In this case, one can do everything globally, since the notions of infinitesimal invariance with respect to the coideal $\mathfrak{k}^{(1,0)}$ and global invariance with respect to the quantum subgroup $U_{q}(n-1) \times U(1)$ coincide. The resulting spherical functions are little $q$-Jacobi polynomials, which are orthogonal with respect to a measure supported on an infinite discrete set (Jackson $q$-integral).

Secondly, the case $n=2(c, d$ arbitrary) has been thoroughly studied in a number of papers. The quantum projective spaces then reduce to quantum spheres, which were introduced globally by Podleś $[\mathrm{P}]$. The zonal spherical functions on these quantum spheres were studied by Mimachi and the second author [NM1], and, in general, by Koornwinder [K2], [K3], who used the infinitesimal method. For the special case $n=2(c=1, d=0)$ we also refer to the papers by Vaksman and Sorbel'man [VS1], Masuda et al. [M], and Koornwinder [K1].

The organization of this paper is as follows. In sections 1 and 2 we collect the necessary facts about the quantum unitary group and $q$-hypergeometric orthogonal polynomials respectively. In section 3 we introduce the coideals $\mathfrak{k}^{(c, d)}$ and study the corresponding quantized algebras of invariant functions and their Plancherel decomposition under the natural transitive $U_{q}(n)$-action. In section 4 we show that the algebras of invariant functions can be constructed by means of a $q$-analogue of the Hopf fibration $S^{2 n-1} \rightarrow \mathbb{C P}^{n-1}$ and establish the link with Vaksman and Korogodsky $[\mathrm{KV}]$. In section 5 we consider the zonal spherical functions and identify them as a (partially discrete) three-parameter family of Askey-Wilson polynomials. We also treat some special cases $(c=0$ or $d=0)$ in which the Askey-Wilson polynomials degenerate to big or little $q$-Jacobi polynomials. In section 6 we present the details of the computation of the radial part of a suitable Casimir operator.

The main results of this paper were announced in $[\mathrm{Dz}]$. The reader is referred to that paper for more background information and a discussion of the results presented here in the context of general higher-rank quantum symmetric spaces $[\mathrm{N}],[\mathrm{NS} 1],[\mathrm{NS} 2],[\mathrm{S} 2]$.

The authors would like to thank T. Sugitani for numerous stimulating discussions and some very useful suggestions. The research for this paper was started at the 
University of Amsterdam. The authors would like to express their gratitude to Professor Tom H. Koornwinder for his hospitality and for providing a stimulating environment in which to do research, the first author as a former temporary member of Professor Koornwinder's research group, the second one as an invited speaker at the Thomas Stieltjes Research Institute during the Concentration Period on Representation Theory and $q$-Special Functions (April-May 1994). The first author would also like to thank the Mittag-Leffler Institute in Stockholm for its hospitality while preparing the final version of this paper.

\section{Preliminaries on the quantum unitary group}

The quantum unitary group $U_{q}(n)$ or its "infinitesimal" version, the quantized universal enveloping algebra $\mathcal{U}_{q}=\mathcal{U}_{q}(\mathfrak{g l}(n))$, have been studied in many papers, for instance $[\mathrm{J}],[\mathrm{Dr} 1],[\mathrm{RTF}],[\mathrm{So}],[\mathrm{NYM}],[\mathrm{N}]$. Our basic reference will be $[\mathrm{N}, \S 1]$. In this section we only recall the barest essentials. Our notation is virtually the same as in $[\mathrm{N}, \S 1]$.

Let us fix $0<q<1$ and $n \geq 2$. Let $V$ be the vector space over $\mathbb{C}$ with canonical basis $\left(v_{i}\right)_{1 \leq i \leq n}$. Our starting point is the invertible $n^{2} \times n^{2}$ matrix $R \in \operatorname{End}(V \otimes V)$ defined by

$$
R:=\sum_{i j} q^{\delta_{i j}} e_{i i} \otimes e_{j j}+\left(q-q^{-1}\right) \sum_{i>j} e_{i j} \otimes e_{j i},
$$

where the $e_{i j} \in \operatorname{End}(V)$ denote the standard matrix units with respect to the basis $\left(v_{i}\right)$. The generators $t_{i j}$ of $\mathcal{A}_{q}=\mathcal{A}_{q}(U(n))$ satisfy the usual commutation relations $R T_{1} T_{2}=T_{2} T_{1} R$ and form a unitary matrix corepresentation of $\mathcal{A}_{q}$.

Let $\mathcal{A}(\mathbb{T}):=\mathbb{C}\left[z_{1}^{ \pm 1}, \ldots, z_{n}^{ \pm 1}\right]$ be the algebra of trigonometric polynomials on the $n$-dimensional real torus $\mathbb{T}$, the Hopf $*$-algebra structure being given by

$$
\Delta\left(z_{i}\right)=z_{i} \otimes z_{i}, \quad \varepsilon\left(z_{i}\right)=1, \quad z_{i}^{*}=z_{i}^{-1} \quad(1 \leq i \leq n) .
$$

$\mathbb{T}$ is naturally identified with the diagonal subgroup of $U_{q}(n)$, the corresponding surjective Hopf $*$-algebra morphism (restriction of functions) being denoted by

$$
{ }_{\mathrm{I} T}: \mathcal{A}_{q} \longrightarrow \mathcal{A}(\mathbb{T}) \text {. }
$$

Let $P=\bigoplus_{1<i<n} \mathbb{Z} \varepsilon_{i}$ denote the weight lattice of $U(n)$. We identify $P$ and $P^{*}=\operatorname{Hom}_{\mathbb{Z}}(P, \mathbb{Z})$ by means of the pairing $\left\langle\varepsilon_{i}, \varepsilon_{j}\right\rangle=\delta_{i j}$. The algebra $\mathcal{U}_{q}$ is generated by the symbols $q^{h}\left(h \in P^{*}\right)$ and $e_{i}, f_{i}(1 \leq i \leq n-1)$ subject to the well-known quantized Weyl-Serre relations. Let $\mathcal{U}_{q}(\mathfrak{h}) \subset \mathcal{U}_{q}$ denote the subalgebra generated by the elements $q^{h}\left(h \in P^{*}\right)$.

We put

$$
R^{+}:=P R P, \quad R^{-}:=R^{-1},
$$

where $P \in \operatorname{End}(V \otimes V)$ is the usual permutation operator. One has the identities $\left(R^{\epsilon}\right)^{-1}=\left(R^{-\epsilon}\right)^{t}(\epsilon= \pm)$. The algebra $\mathcal{U}_{q}$ is also generated by the so-called $L$ operators $L_{i j}^{+}, L_{i j}^{-} \in \mathcal{U}_{q}$ (cf. [J], [RTF], [N]) subject to the relations

$$
R^{+} L_{1}^{\epsilon} L_{2}^{\epsilon}=L_{2}^{\epsilon} L_{1}^{\epsilon} R^{+}(\epsilon= \pm), \quad R^{+} L_{1}^{+} L_{2}^{-}=L_{2}^{-} L_{1}^{+} R^{+},
$$

where $L^{ \pm}:=\left(L_{i j}^{ \pm}\right), L_{1}^{ \pm}:=L^{ \pm} \otimes$ id, etc. In particular,

$$
q^{h} L_{i j}^{ \pm} q^{-h}=q^{\left\langle h, \varepsilon_{j}-\varepsilon_{i}\right\rangle} L_{i j}^{ \pm}, \quad q^{h} S\left(L_{i j}^{ \pm}\right) q^{-h}=q^{\left\langle h, \varepsilon_{j}-\varepsilon_{i}\right\rangle} S\left(L_{i j}^{ \pm}\right)(1 \leq i, j \leq n) .
$$


The $L_{i j}^{ \pm}$can be viewed as quantum analogues of arbitrary root vectors in $\mathfrak{g l}(n)$. The matrices $L^{+}$and $L^{-}$are upper and lower triangular respectively.

The Hopf $*$-algebra structure on $\mathcal{U}_{q}$ is uniquely determined by

$$
\Delta\left(L_{i j}^{ \pm}\right)=\sum_{k} L_{i k}^{ \pm} \otimes L_{k j}^{ \pm}, \quad \varepsilon\left(L_{i j}^{ \pm}\right)=\delta_{i j}, \quad\left(L_{i j}^{ \pm}\right)^{*}=S\left(L_{j i}^{\mp}\right) \quad(1 \leq i, j \leq n) .
$$

The action of the involution $\tau=* \circ S: \mathcal{U}_{q} \rightarrow \mathcal{U}_{q}$ on the generators is given by

$$
\tau\left(L_{i j}^{ \pm}\right)=L_{j i}^{\mp} \quad(1 \leq i, j \leq n) .
$$

The cone $P^{+} \subset P$ of dominant weights by definition consists of all weights $\lambda=\sum_{k} \lambda_{k} \varepsilon_{k} \in P$ such that $\lambda_{1} \geq \ldots \geq \lambda_{n}$. There is the usual parametrization $\lambda \mapsto V(\lambda)$ of irreducible $P$-weighted finite-dimensional left $\mathcal{U}_{q}$-modules by dominant weights (cf. $[\mathrm{L}],[\mathrm{R}])$. Recall that a highest weight vector of a left $\mathcal{U}_{q}$-module $W$ is by definition annihilated by the $L_{i j}^{-}$or, equivalently, by the $S\left(L_{i j}^{-}\right)(i>j)$. All finitedimensional $P$-weighted left $\mathcal{U}_{q}$-modules are completely reducible and unitarizable.

Weights of right $\mathcal{U}_{q}$-modules are defined in the obvious way, highest weight vectors being killed by definition by the $L_{i j}^{+}$or the $S\left(L_{i j}^{+}\right)(i>j)$. Given a left $\mathcal{U}_{q}$-module $W$, one defines a right $\mathcal{U}_{q}$-module structure on the conjugate vector space $W^{\circ}$ by putting

$$
v \cdot u:=u^{*} \cdot v \quad\left(v \in W, u \in \mathcal{U}_{q}\right) .
$$

The assignment $W \mapsto W^{\circ}$ is a 1-1 correspondence between left and right $\mathcal{U}_{q}$-modules preserving weight vectors, weights, and highest weights.

There exists a unique algebra homomorphism $\rho_{V}: \mathcal{U}_{q} \rightarrow \operatorname{End}(V)$ such that

$$
R^{ \pm}=\sum_{i j} e_{i j} \otimes \rho_{V}\left(L_{i j}^{ \pm}\right), \quad\left(R^{ \pm}\right)^{-1}=\sum_{i j} e_{i j} \otimes \rho_{V}\left(S\left(L_{i j}^{ \pm}\right)\right) .
$$

The corresponding representation $V$ is called the vector representation and has highest weight $\varepsilon_{1}$. The elements $q^{h} \in \mathcal{U}_{q}(\mathfrak{h})$ act on $V$ as diagonal matrices with respect to the basis $\left(v_{i}\right)$.

By means of the natural Hopf $*$-algebra duality $\langle\cdot, \cdot\rangle$ between $\mathcal{U}_{q}$ and $\mathcal{A}_{q}$, one can identify $\mathcal{A}_{q}$ with a subspace of the algebraic linear dual of $\mathcal{U}_{q}$. There is an induced Hopf $*$-algebra duality between $\mathcal{U}_{q}(\mathfrak{h})$ and $\mathcal{A}(\mathbb{T})$ such that

$$
\left\langle q^{h}, z^{\lambda}\right\rangle:=q^{\langle h, \lambda\rangle}, \quad z^{\lambda}=z_{1}^{\lambda_{1}} \cdots z_{n}^{\lambda_{n}} \quad\left(h \in P^{*}, \lambda=\sum_{k} \lambda_{k} \varepsilon_{k} \in P\right) .
$$

The duality naturally turns $\mathcal{A}_{q}$ into a $\mathcal{U}_{q}$-bimodule with two-sided $\mathcal{U}_{q}$-symmetry. Recall that transposition defines a natural right $\mathcal{U}_{q}$-module structure on the linear dual $\operatorname{Hom}(V, \mathbb{C})=V^{*}$. The mapping

$$
V \otimes \operatorname{Hom}(V, \mathbb{C}) \rightarrow \mathcal{A}_{q}, \quad v_{i} \otimes v_{j}^{*} \mapsto t_{j i}
$$

is an (injective) $\mathcal{U}_{q}$-bimodule homomorphism. More generally, one has the following decomposition of $\mathcal{A}_{q}$ into irreducible $\mathcal{U}_{q}$-bimodules:

$$
\mathcal{A}_{q}=\bigoplus_{\lambda \in P^{+}} V(\lambda) \otimes \operatorname{Hom}(V(\lambda), \mathbb{C}) .
$$

Here the subspace $W(\lambda):=V(\lambda) \otimes \operatorname{Hom}(V(\lambda), \mathbb{C}) \subset \mathcal{A}_{q}$ is spanned by the matrix coefficients of the (co-)representation $V(\lambda)$. Note that $\operatorname{Hom}(V(\lambda), \mathbb{C})$ is isomorphic with $V(\lambda)^{\circ}$, although the isomorphism is not canonical. The decomposition (1.13) 
can also be characterized as the simultaneous eigenspace decomposition of $\mathcal{A}_{q}$ with respect to the natural action of the center $\mathcal{Z} \mathcal{U}_{q} \subset \mathcal{U}_{q}$.

Let $h: \mathcal{A}_{q} \rightarrow \mathbb{C}$ denote the Haar functional on $\mathcal{A}_{q}$ (cf. [W], [SV], [NYM], [DK2]). Then $\langle a, b\rangle:=h\left(b^{*} a\right)$ defines a positive definite inner product on $\mathcal{A}_{q}$ with respect to which the subspaces $W(\lambda) \subset \mathcal{A}_{q}$ are mutually orthogonal (Schur orthogonality).

\section{Preliminaries on $q$-Hypergeometric orthogonal polynomials}

Let $0<q<1$. The so-called $q$-shifted factorials are defined as

$$
\begin{aligned}
(a ; q)_{n} & :=\prod_{k=0}^{n-1}\left(1-a q^{k}\right),\left(a_{1}, \ldots, a_{s} ; q\right)_{n}:=\prod_{j=1}^{s}\left(a_{j} ; q\right)_{n}, \\
(a ; q)_{\infty} & :=\lim _{n \rightarrow \infty}(a ; q)_{n},
\end{aligned}
$$

and the $q$-hypergeometric series $s+1 \varphi_{s}$ as

$$
{ }_{s+1} \varphi_{s}\left[\begin{array}{c}
a_{1}, \ldots, a_{s+1} \\
b_{1}, \ldots, b_{s}
\end{array} ;, z\right]:=\sum_{k=0}^{\infty} \frac{\left(a_{1}, \ldots, a_{s+1} ; q\right)_{k} z^{k}}{\left(b_{1}, \ldots, b_{s} ; q\right)_{k}(q ; q)_{k}} .
$$

We shall only deal with the special case of $(2.2)$ when $a_{1}=q^{-n}\left(n \in \mathbb{Z}_{+}\right)$. The series then is terminating, i.e. for $j>n$ the $j$-th term vanishes. In this case, it is always tacitly assumed that $b_{1}, \ldots, b_{s} \notin\left\{1, q^{-1}, \ldots, q^{-n+1}\right\}$. Further details about $q$-hypergeometric functions can be found in [GR, Ch. 1].

Consider the Laurent polynomial ring $\mathbb{C}\left[z^{ \pm 1}\right]$ in the variable $z$, and put $x:=$ $\frac{1}{2}\left(z+z^{-1}\right)$. The Askey-Wilson polynomials (cf. [AW]) are the polynomials in the variable $x$ given by

$$
\begin{aligned}
& p_{n}(x ; a, b, c, d \mid q) \\
& \quad:=a^{-n}(a b, a c, a d ; q)_{n} \cdot{ }_{4} \varphi_{3}\left[\begin{array}{c}
q^{-n}, q^{n-1} a b c d, a z, a z^{-1} \\
a b, a c, a d
\end{array} ; q, q\right] .
\end{aligned}
$$

They are symmetric in $a, b, c, d$. It is sometimes convenient to write the ${ }_{4} \varphi_{3}$ factor in (2.3) as $r_{n}(x)=R_{n}(z)$. Depending on the value of $a, b, c, d$, Askey-Wilson polynomials are orthogonal with respect to a positive orthogonality measure consisting of a continuous and a discrete part. To be more precise, one has the following result (cf. [AW, Thm. 2.5]):

Proposition 2.1. Assume that (i) $a, b, c, d$ are real, or, if complex, appear in conjugate pairs, and (ii) the pairwise products of $a, b, c, d$ are not $\geq 1$. Then the Askey-Wilson polynomials $p_{n}(x)$ are mutually orthogonal with respect to the positive definite inner product on $\mathbb{C}[x]$ defined by

$$
\begin{aligned}
\langle P, Q\rangle:=\frac{1}{2 \pi i} \int_{z \in \mathcal{C}} P(x) & \overline{Q(x)} w(z ; a, b, c, d ; q) \frac{d z}{z} \\
& +\sum_{e, k} P\left(e_{k}\right) \overline{Q\left(e_{k}\right)} w_{k}(e, f, g, h) \quad(P, Q \in \mathbb{C}[x]) .
\end{aligned}
$$

Here integration is along the unit circle $\mathcal{C}$ in counterclockwise direction, the summation runs over all $e \in\{a, b, c, d\}$ such that $|e|>1$ and all $k \in \mathbb{Z}_{+}$such that $\left|e q^{k}\right|>1$. Moreover, the following notation is used:

$$
w(z ; a, b, c, d ; q):=\frac{\left(z^{2}, z^{-2} ; q\right)_{\infty}}{(a z, a / z, b z, b / z, c z, c / z, d z, d / z ; q)_{\infty}},
$$




$$
\begin{gathered}
w_{k}(e, f, g, h):=\frac{\left(e^{-2} ; q\right)_{\infty}}{(q, e f, f / e, e g, g / e, e h, h / e ; q)_{\infty}} \\
\times \frac{\left(e^{2}, e f, e g, e h ; q\right)_{k}}{(q, e q / f, e q / g, e q / h ; q)_{k}} \frac{\left(1-e^{2} q^{2 k}\right)}{\left(1-e^{2}\right)}\left(\frac{q}{e f g h}\right)^{k}, \\
e_{k}:=\left(e q^{k}+e^{-1} q^{-k}\right) / 2,
\end{gathered}
$$

with $(e, f, g, h)$ any permutation of $(a, b, c, d)$.

One can calculate an explicit expression for the norm of the Askey-Wilson polynomials $p_{n}(x ; a, b, c, d \mid q)$ with respect to the inner product (2.4) (cf. [AW, Thm. 2.5]). We shall only need the value for $p_{0}=1$ (cf. [AW, Thm. 2.1]):

$$
\langle 1,1\rangle=\frac{2(a b c d ; q)_{\infty}}{(q, a b, a c, a d, b c, b d, c d ; q)_{\infty}} .
$$

For certain special values of the parameters, e.g. $\max (|a|,|b|,|c|,|d|)<1$, the discrete part in (2.4) becomes void. The orthogonality measure then is absolutely continuous.

The Laurent polynomials $R_{n}(z)$ in the variable $z$ satisfy the following secondorder $q$-difference equation:

$$
\begin{aligned}
& A(z ; q)\left(R_{n}(q z)-R_{n}(z)\right)+A\left(z^{-1} ; q\right)\left(R_{n}\left(q^{-1} z\right)-R_{n}(z)\right) \\
& \quad=-\left(1-q^{-n}\right)\left(1-q^{n-1} a b c d\right) R_{n}(z),
\end{aligned}
$$

where

$$
A(z ; q):=\frac{(1-a z)(1-b z)(1-c z)(1-d z)}{\left(1-z^{2}\right)\left(1-q z^{2}\right)} \quad(a, b, c, d \in \mathbb{C}) .
$$

Any symmetric Laurent polynomial $f(z)$ that is of degree $\leq n$ when viewed as a polynomial in $x$ and satisfies (2.6) is a constant multiple of $R_{n}(z)$.

There are the so-called big $q$-Jacobi polynomials (cf. [AA2]):

$$
P_{n}^{(\alpha, \beta)}(x ; c, d: q):={ }_{3} \varphi_{2}\left[\begin{array}{c}
q^{-n}, q^{n+\alpha+\beta+1}, q^{\alpha+1} x / c \\
q^{\alpha+1},-q^{\alpha+1} d / c
\end{array} ; q, q\right] .
$$

If $c, d>0$ and $\alpha, \beta>-1$, then the polynomials $P_{n}^{(\alpha, \beta)}(x ; c, d: q)$ are orthogonal with respect to a positive orthogonality measure supported on the infinite discrete set

$$
\left\{c q^{k} \mid k \in \mathbb{Z}_{+}\right\} \cup\left\{-d q^{k} \mid k \in \mathbb{Z}_{+}\right\} \subset[-d, c] .
$$

A special case of the big $q$-Jacobi polynomials $(c=1, d=0)$ are the so-called little $q$-Jacobi polynomials (cf. [AA1]):

$$
p_{n}^{(\alpha, \beta)}(x: q):={ }_{2} \varphi_{1}\left(q^{-n}, q^{\alpha+\beta+n+1} ; q^{\alpha+1} ; q ; q x\right) .
$$

For $\alpha, \beta>-1$ they are orthogonal with respect to a positive orthogonality measure supported on the infinite discrete set $\left\{q^{k} \mid k \in \mathbb{Z}_{+}\right\} \subset[0,1]$.

Both big and little $q$-Jacobi polynomials may be characterized as the polynomial eigenfunctions of a certain second-order $q$-difference operator with rational coefficients and depending on the parameters $\alpha, \beta$ (and $c, d$ in the big $q$-Jacobi case).

Recall the notation $r_{n}(x)=r_{n}(x ; a, b, c, d \mid q)$ for the ${ }_{4} \varphi_{3}$ factor in (2.3). Little and big $q$-Jacobi polynomials can be recovered from the Askey-Wilson polynomials $r_{n}$ by a suitable limit transition (cf. [K3, Prop. 6.1, 6.3]): 
Proposition 2.2. Let the big q-Jacobi polynomials be denoted as in (2.8). Then

$$
\begin{aligned}
\lim _{a \rightarrow 0} r_{n}( & \frac{q^{\frac{1}{2}} x}{2 a(c d)^{\frac{1}{2}}} ; q^{\alpha+\frac{1}{2}} a(d / c)^{\frac{1}{2}}, q^{\frac{1}{2}} a^{-1}(c / d)^{\frac{1}{2}}, \\
& \left.\quad-q^{\frac{1}{2}} a^{-1}(d / c)^{\frac{1}{2}},-q^{\beta+\frac{1}{2}} a(c / d)^{\frac{1}{2}} \mid q\right)=P_{n}^{(\alpha, \beta)}(x ; c, d: q) .
\end{aligned}
$$

Proposition 2.3. Let the little q-Jacobi polynomials be denoted as in (2.9). Then

$$
\lim _{a \rightarrow 0} r_{n}\left(\frac{q^{\frac{1}{2}} x}{ \pm 2 a^{2}} ; \pm q^{\alpha+\frac{1}{2}} a^{2}, \pm q^{\frac{1}{2}} a^{-2}, \mp q^{\frac{1}{2}}, \mp q^{\beta+\frac{1}{2}} \mid q\right)=\frac{\left(q^{\beta+1} ; q\right)_{n}}{\left(q^{-n-\alpha} ; q\right)_{n}} p_{n}^{(\beta, \alpha)}( \pm x: q) .
$$

As follows from Proposition 2.1 after a suitable transformation of variables (cf. [K3, Remark 6.6]), for $\alpha, \beta>-1, c, d>0$ and $a$ small enough (and positive), the orthogonal polynomials in $x$ on the left-hand side of the displayed equation in Proposition 2.2 (after the limit sign) have continuous mass on the interval $\left[-2 a(c d / q)^{\frac{1}{2}}, 2 a(c d / q)^{\frac{1}{2}}\right]$ and discrete mass on the two finite sets

$$
\left\{c q^{k}+a^{2} d q^{-k-1} \mid k \in \mathbb{Z}_{+}, q^{k}>a(q c / d)^{-\frac{1}{2}}\right\}
$$

and

$$
\left\{-d q^{k}-a^{2} c q^{-k-1} \mid k \in \mathbb{Z}_{+}, q^{k}>a(q d / c)^{-\frac{1}{2}}\right\} .
$$

When $a \rightarrow 0$, the continuous mass interval shrinks to $\{0\}$, while the two discrete sets tend to the support of the orthogonality measure of the big $q$-Jacobi polynomials. A similar remark applies to little $q$-Jacobi polynomials.

\section{A FAMILY OF TWO-SIDED COIDEALS}

Let us fix real numbers $c, d \geq 0$ such that $(c, d) \neq(0,0)$. The subspace $\mathfrak{k}^{(c, d)} \subset \mathcal{U}_{q}$ is by definition spanned by the following elements:

$$
\begin{aligned}
\text { (i) } & L_{11}^{+}-L_{n n}^{-}, L_{11}^{-}-L_{n n}^{+}, \\
\text {(ii) } & \sqrt{c} L_{1 k}^{+}+\sqrt{d} L_{n k}^{-} \quad(2 \leq k \leq n-1), \\
\text { (iii) } & \sqrt{d} L_{k n}^{+}+\sqrt{c} L_{k 1}^{-} \quad(2 \leq k \leq n-1), \\
\text { (iv) } & L_{i j}^{+}, L_{j i}^{-} \quad(2 \leq i<j \leq n-1), \\
\text { (v) } & L_{i i}^{+}-L_{i i}^{-} \quad(2 \leq i \leq n-1), \\
\text { (vi) } & \sqrt{c d} L_{1 n}^{+}-\sqrt{c d} L_{n 1}^{-}-(c-d)\left(L_{11}^{+}-L_{11}^{-}\right) .
\end{aligned}
$$

We remark that the subspace $\mathfrak{k} \subset \mathcal{U}_{q}$ only depends on the ratio of the numbers $c$ and $d$. In fact, it will be convenient to introduce a parameter $\sigma \in \mathbb{R} \cup\{ \pm \infty\}$ by setting

$$
q^{\sigma}=\sqrt{\frac{d}{c}}(c, d>0), \quad \sigma=\infty \quad(d=0), \quad \sigma=-\infty \quad(c=0) .
$$

Then $\mathfrak{k}^{(c, d)}$ only depends on $\sigma$ and we write $\mathfrak{k}^{\sigma}:=\mathfrak{k}^{(c, d)}$.

In the remainder of this paper, whenever we write $\mathfrak{k}^{\sigma}$ or $\mathfrak{k}^{(c, d)}$, it is tacitly assumed that $-\infty \leq \sigma \leq \infty$ and $c, d \geq 0,(c, d) \neq(0,0)$, unless explicitly mentioned otherwise.

Proposition 3.1. The subspace $\mathfrak{k}^{\sigma} \subset \mathcal{U}_{q}$ is a $\tau$-invariant two-sided coideal. 
Proof. It follows from (1.8) that $\tau$ permutes the elements listed in (3.1) (up to a scalar multiple). Hence $\mathfrak{k}^{\sigma}$ is $\tau$-invariant. The property $\varepsilon\left(\mathfrak{k}^{\sigma}\right)=0$ is a direct consequence of (1.7). A straightforward computation using (1.7) shows that $\Delta\left(\mathfrak{k}^{\sigma}\right) \subset \mathfrak{k}^{\sigma} \otimes \mathcal{U}_{q}+\mathcal{U}_{q} \otimes \mathfrak{k}^{\sigma}$. We leave the details to the reader.

We now proceed to study $\mathfrak{k}^{\sigma}$-fixed and $\left(\mathfrak{k}^{*}\right)^{\sigma}$-fixed vectors in finite-dimensional representations of $\mathcal{U}_{q}$. Let $W$ be a left $\mathcal{U}_{q}$-module. A vector $v \in W$ is called invariant w.r.t. an element $u \in \mathcal{U}_{q}$ if $u \cdot v=\varepsilon(u) v$. In particular, the subspace $W_{\mathfrak{k} \sigma} \subset W$ of $\mathfrak{k}^{\sigma}$-fixed vectors is defined as

$$
W_{\mathfrak{k}^{\sigma}}:=\left\{v \in W \mid \mathfrak{k}^{\sigma} \cdot v=0\right\} .
$$

It is obvious how to define $\mathfrak{k}^{\sigma}$-fixed vectors for right $\mathcal{U}_{q}$-modules. Note that a vector $v$ in a right $\mathcal{U}_{q}$-module $W$ is $\mathfrak{k}^{\sigma}$-fixed if and only if $v$ is $\left(\mathfrak{k}^{\sigma}\right)^{*}$-fixed as an element of the left $\mathcal{U}_{q}$-module $W^{\circ}$.

Assuming that $\sigma$ is finite, we have the following crucial result:

Proposition 3.2. Suppose $\sigma$ is finite and let $\mathfrak{k}$ denote either $\mathfrak{k}^{\sigma}$ or $\left(\mathfrak{k}^{*}\right)^{\sigma}$. Suppose $\lambda \in P^{+}$. If $v \in V(\lambda)$ is a non-zero $\mathfrak{k}$-fixed vector, then the highest weight component of $v$ is non-zero.

Proof. The proof is similar to that of [N, Lemma 3.2]. Suppose that $0 \neq v \in V(\lambda)$ is $\mathfrak{k}^{\sigma}$-fixed and write $v$ as a sum $v=\sum_{\mu \in P} v_{\mu}$ of weight vectors. Recall (cf. (1.6)) that $L_{i j}^{ \pm}$has weight $\varepsilon_{j}-\varepsilon_{i}$ in $\mathcal{U}_{q}$. This implies that $L_{i j}^{ \pm} \cdot v_{\mu} \in V(\lambda)$ has weight $\mu+\varepsilon_{j}-\varepsilon_{i}$. Let $\leq$ denote the lexicographic order on $P$ with respect to the $\mathbb{Z}$-basis $\left(\varepsilon_{i}\right)$. One reads off from (3.1) that, for any $i<j$, there is an element $X_{i j} \in \mathfrak{k}^{\sigma}$ whose leading term (w.r.t. $\leq$ ) is equal to $L_{j i}^{-}$. Now let $\mu_{0} \in P$ be the greatest element $\mu \in P$ (w.r.t. $\leq$ ) such that $v_{\mu} \neq 0$. Then $L_{j i}^{-} \cdot v_{\mu_{0}}$ is the component of weight $\mu_{0}+\varepsilon_{j}-\varepsilon_{i}$ in $X_{i j} \cdot v$. But $X_{i j} \cdot v=0$, since $X_{i j} \in \mathfrak{k}^{\sigma}$. Hence $L_{i j}^{-} \cdot v_{\mu_{0}}=0$. In other words, $v_{\mu_{0}}$ is a non-zero highest weight vector in $V(\lambda)$, which forces $\mu_{0}=\lambda$. One can prove the corresponding statement for $\left(\mathfrak{k}^{*}\right)^{\sigma}$ in a completely analogous way using (1.7) and the fact that a highest weight vector $v$ is characterized among weight vectors by the condition $S\left(L_{i j}^{-}\right) \cdot v=0(1 \leq j<i \leq n)$.

Corollary 3.3. Suppose $\sigma$ is finite and let $\mathfrak{k}$ denote either $\mathfrak{k}^{\sigma}$ or $\left(\mathfrak{k}^{*}\right)^{\sigma}$. Suppose $\lambda \in P^{+}$. The subspace $V(\lambda)_{\mathfrak{k}}$ of $\mathfrak{k}$-fixed vectors is at most one-dimensional.

Proof. This is a direct consequence of the preceding proposition (cf. [N, §3.1]).

Corollary 3.3 can also be expressed by saying that $\left(\mathcal{U}_{q}, \mathfrak{k}^{\sigma}\right)$ resp. $\left(\mathcal{U}_{q},\left(\mathfrak{k}^{*}\right)^{\sigma}\right)$ satisfy the Gelfand pair property. We call $V(\lambda)\left(\lambda \in P^{+}\right)$spherical (with respect to $\mathfrak{k}^{\sigma}$ ) if it has non-zero $\mathfrak{k}^{\sigma}$-fixed vectors.

In order to determine the spherical representations we need some explicit information about the vector representation $V$ and its contragredient $V^{*}$. One deduces from (1.10) that the action of the $L_{i j}^{ \pm}$and the $S\left(L_{i j}^{ \pm}\right)$on the basis vectors $v_{i} \in V$ is given by the following explicit formulae:

$$
\begin{aligned}
& L_{i i}^{\epsilon} \cdot v_{k}=q^{\epsilon \delta_{i k}} v_{k}, \quad S\left(L_{i i}^{\epsilon}\right) \cdot v_{k}=q^{-\epsilon \delta_{i k}} v_{k}(\epsilon= \pm), \\
& L_{i j}^{ \pm} \cdot v_{k}= \pm\left(q-q^{-1}\right) \delta_{i k} v_{j}, \quad S\left(L_{i j}^{ \pm}\right) \cdot v_{k}=\mp\left(q-q^{-1}\right) \delta_{i k} v_{j}(i \lessgtr j) .
\end{aligned}
$$

The vector $v_{i}(1 \leq i \leq n)$ has weight $\varepsilon_{i}$. The highest weight vector is $v_{1}$. Recall that the action of $\mathcal{U}_{q}$ on the contragredient module $V^{*}$ is given by

$$
\left(u \cdot v^{*}\right)(v)=v^{*}(S(u) \cdot v) \quad\left(v \in V, v^{*} \in V^{*}\right) .
$$


Let $\left(v_{i}^{*}\right)$ denote the dual basis of $V^{*}$. The action of the $L_{i j}^{ \pm}$and the $S\left(L_{i j}^{ \pm}\right)$on the basis vectors $v_{k}^{*}$ is given by

$$
\begin{aligned}
& L_{i i}^{\epsilon} \cdot v_{k}^{*}=q^{-\epsilon \delta_{i k}} v_{k}^{*}, \quad S\left(L_{i i}^{\epsilon}\right) \cdot v_{k}^{*}=q^{\epsilon \delta_{i k}} v_{k}^{*}(\epsilon= \pm), \\
& L_{i j}^{ \pm} \cdot v_{k}^{*}=\mp\left(q-q^{-1}\right) \delta_{j k} v_{i}^{*}, S\left(L_{i j}^{ \pm}\right) \cdot v_{k}^{*}= \pm q^{2(j-i)}\left(q-q^{-1}\right) \delta_{j k} v_{i}^{*}(i \lessgtr j) .
\end{aligned}
$$

To compute the action of the $S\left(L_{i j}^{ \pm}\right)$in (3.6) one needs the fact (cf. [Dr2, Prop. 2.1], [RTF, Thm. 11], [N, (1.15)]) that the square of the antipode $S^{2}: \mathcal{U}_{q} \rightarrow \mathcal{U}_{q}$ is given by

$$
S^{2}(u)=q^{-2 \rho} u q^{2 \rho}, \quad \rho:=\sum_{k=1}^{n}(n-k) \varepsilon_{k} \in P .
$$

The vector $v_{i}^{*}(1 \leq i \leq n)$ has weight $-\varepsilon_{i}$. The highest weight vector is $v_{n}^{*}$. The $t_{i j}^{*}=S\left(t_{j i}\right)$ are the coefficients of $V^{*}$ with respect to the basis $\left(v_{i}^{*}\right)$.

The tensor product $V^{*} \otimes V$ has the following irreducible decomposition:

$$
V^{*} \otimes V \cong V(0) \oplus V\left(\varepsilon_{1}-\varepsilon_{n}\right) .
$$

Here the subspace $V(0)$ is spanned by the element $\sum_{k} q^{2(n-k)} v_{k}^{*} \otimes v_{k}$. The subspace $V\left(\varepsilon_{1}-\varepsilon_{n}\right)$ is spanned by the linearly independent vectors

$$
v_{i}^{*} \otimes v_{j} \quad(1 \leq i \neq j \leq n), \quad v_{i}^{*} \otimes v_{i}-v_{i+1}^{*} \otimes v_{i+1} \quad(1 \leq i \leq n-1) .
$$

The vector $v_{i}^{*} \otimes v_{j}(1 \leq i, j \leq n)$ has weight $\varepsilon_{j}-\varepsilon_{i}$. The highest weight vector in $V\left(\varepsilon_{1} \otimes \varepsilon_{n}\right)$ is $v_{n}^{*} \otimes v_{1}$. All these statements can be easily deduced from (3.4), (3.6), and (1.7).

Theorem 3.4. Let $\sigma$ be finite and let $\mathfrak{k}$ denote either $\mathfrak{k}^{\sigma}$ or $\left(\mathfrak{k}^{*}\right)^{\sigma}$. For any $\lambda \in P^{+}$, the representation $V(\lambda)$ has non-zero $\mathfrak{k}$-fixed vectors if and only if $\lambda=l\left(\varepsilon_{1}-\varepsilon_{n}\right)$ for some $l \in \mathbb{Z}_{+}$.

Proof. We first prove the "only if" part of the statement. Let $v \in V(\lambda)$ be a nonzero $\mathfrak{k}$-fixed vector with highest weight component $v_{\lambda} \neq 0$. For any $2 \leq i \leq n-1$ one has $0=\left(L_{i i}^{+}-L_{i i}^{-}\right) \cdot v_{\lambda}=\left(q^{\left\langle\lambda, \varepsilon_{i}\right\rangle}-q^{-\left\langle\lambda, \varepsilon_{i}\right\rangle}\right) v_{\lambda}$. Hence $\lambda \in \mathbb{Z} \varepsilon_{1} \oplus \mathbb{Z} \varepsilon_{n}$. On the other hand, $\left(L_{11}^{+}-L_{n n}^{-}\right) \cdot v_{\lambda}=0$. This implies $q^{\left\langle\lambda, \varepsilon_{1}\right\rangle}=q^{-\left\langle\lambda, \varepsilon_{n}\right\rangle}$, hence $\lambda=l\left(\varepsilon_{1}-\varepsilon_{n}\right)$ for some $l \in \mathbb{Z}_{+}$. To prove the "if" part of the statement we first exhibit a $\mathfrak{k}$-fixed vector in the "lowest" spherical representation $V\left(\varepsilon_{1}-\varepsilon_{n}\right)$. In the representation $V^{*} \otimes V$ we have the following $\mathfrak{k}$-fixed vector $w_{\mathfrak{k}}$ :

$$
\begin{aligned}
w_{\mathfrak{k} \sigma} & :=\sqrt{c d} v_{1}^{*} \otimes v_{n}+\sqrt{c d} v_{n}^{*} \otimes v_{1}+q d v_{1}^{*} \otimes v_{1}+q^{-1} c v_{n}^{*} \otimes v_{n}, \\
w_{\left(\mathfrak{k}^{*}\right)^{\sigma}} & :=q^{2(n-1)} \sqrt{c d} v_{1}^{*} \otimes v_{n}+\sqrt{c d} v_{n}^{*} \otimes v_{1}+q^{2(n-1)} d v_{1}^{*} \otimes v_{1}+c v_{n}^{*} \otimes v_{n} .
\end{aligned}
$$

One verifies that $w_{\mathfrak{k}}$ is $\mathfrak{k}$-fixed by means of a straightforward computation using (3.4) and (3.6). Since the highest weight term in $v_{\mathfrak{k}}$ is $\sqrt{c d} v_{n}^{*} \otimes v_{1} \neq 0$, the component of $v_{\mathfrak{k}}$ in $V\left(\varepsilon_{1}-\varepsilon_{n}\right)$ is non-zero and obviously k-fixed. To prove the "if" part in general, let us first remark that the vector $\left(v_{n}^{*} \otimes v_{1}\right)^{\otimes l} \in\left(V^{*} \otimes V\right)^{\otimes l}$ is a highest weight vector of weight $l\left(\varepsilon_{1}-\varepsilon_{n}\right)$ occurring with multiplicity 1 inside $\left(V^{*} \otimes V\right)^{\otimes l}$. It generates a $\mathcal{U}_{q}$-submodule isomorphic with $V\left(l\left(\varepsilon_{1}-\varepsilon_{n}\right)\right)$. The component of weight $l\left(\varepsilon_{1}-\varepsilon_{n}\right)$ in $v_{\mathfrak{k}}^{\otimes l}$ is equal to $(c d)^{l / 2}\left(v_{n}^{*} \otimes v_{1}\right)^{\otimes l}$, which is obviously non-zero. This proves that there exists a non-zero $\mathfrak{k}$-fixed vector in $V\left(l\left(\varepsilon_{1}-\varepsilon_{n}\right)\right)$. 
The analysis of the spherical representations for $\sigma= \pm \infty$ proceeds along rather different lines, since there is no analogue for Proposition 3.2. There is a natural embedding of Lie algebras

$$
\mathfrak{g l}(n-1) \oplus \mathfrak{g l}(1) \hookrightarrow \mathfrak{g l}(n), \quad(X, \xi) \mapsto\left(\begin{array}{cc}
X & 0 \\
0 & \xi
\end{array}\right) .
$$

This mapping arises from a natural embedding of the Dynkin diagram of root system $A_{n-2}$ into that of $A_{n-1}$. Since the definition of $\mathcal{U}_{q}(\mathfrak{g l}(n))$ is entirely in terms of generators corresponding to simple roots and relations involving only the Cartan integers, there is an analogue of (3.11) for quantized universal enveloping algebras. To be more precise, by abuse of notation let $U(\mathfrak{g l}(1))$ denote the commutative algebra generated by the symbols $\zeta^{ \pm 1}$ subject to the relation $\zeta \zeta^{-1}=1$. There is a unique Hopf $*$-algebra structure on $U(\mathfrak{g l}(1))$ such that

$$
\Delta\left(\zeta^{ \pm 1}\right)=\zeta^{ \pm 1} \otimes \zeta^{ \pm 1}, \quad \varepsilon\left(\zeta^{ \pm 1}\right)=1, \quad\left(\zeta^{ \pm 1}\right)^{*}=\zeta^{ \pm 1} .
$$

There is a natural injective Hopf $*$-algebra homomorphism

$$
\mathcal{U}_{q}(\mathfrak{k}):=\mathcal{U}_{q}(\mathfrak{g l}(n-1)) \otimes U(\mathfrak{g l}(1)) \hookrightarrow \mathcal{U}_{q}(\mathfrak{g l}(n))
$$

sending $1 \otimes \zeta^{ \pm 1}$ to $q^{ \pm \varepsilon_{n}}$ and $L_{i j}^{ \pm} \otimes 1$ to $L_{i j}^{ \pm}(1 \leq i, j \leq n-1)$. Henceforth, we identify $\mathcal{U}_{q}(\mathfrak{k})$ with its image under this mapping. It is straightforward to check that the notions of invariance w.r.t. $\mathcal{U}_{q}(\mathfrak{k})$ and $\mathfrak{k}^{\infty}$ coincide. The same is true for $(\mathfrak{k})^{*}$, since $\mathcal{U}_{q}(\mathfrak{k})$ is obviously $*$-invariant.

Dual to (3.13) there is a natural surjective Hopf $*$-algebra isomorphism

$$
\mathcal{A}_{q}(U(n)) \rightarrow \mathcal{A}_{q}(U(n-1)) \otimes \mathcal{A}(U(1))=: \mathcal{A}_{q}(K) .
$$

Here $\mathcal{A}(U(1))$ is the algebra of trigonometric polynomials on the one-dimensional real torus $U(1)$ (cf. (1.2)).

The natural notion of invariance w.r.t. $\mathcal{A}_{q}(K)$ coincides with $\mathcal{U}_{q}(\mathfrak{k})$-invariance (cf. [DK1, Prop. 1.12]) and hence with $\mathfrak{k}^{\infty}$-invariance. $\mathcal{A}_{q}(K)$-fixed vectors for finite-dimensional corepresentations of $\mathcal{A}_{q}$ have essentially been analysed in [NYM, $\S 4]$. It can be shown that for finite $\sigma$ there is no quantum subgroup corresponding to the coideal $\mathfrak{k}^{\sigma}$.

Remark 3.5. There is a similar picture in the case $\sigma=-\infty$. Instead of (3.11) one starts with the embedding

$$
\mathfrak{g l}(1) \oplus \mathfrak{g l}(n-1) \hookrightarrow \mathfrak{g l}(n), \quad(\xi, X) \mapsto\left(\begin{array}{cc}
\xi & 0 \\
0 & X
\end{array}\right) .
$$

This embedding is conjugate to (3.11) by the Lie algebra automorphism of $\mathfrak{g l}(n)$ induced by the only non-trivial automorphism of the Dynkin diagram of $A_{n-1}$. Since there are corresponding (dual) Hopf $*$-algebra automorphisms of $\mathcal{U}_{q}$ and $\mathcal{A}_{q}$, every statement about $\mathfrak{k}^{\infty}$-invariance can immediately be translated into a statement about $\mathfrak{k}^{-\infty}$-invariance. In the remainder of this paper, we shall therefore usually confine ourselves to proving statements for $\sigma=\infty$ and leave it to the reader to verify the corresponding statement for $\sigma=-\infty$.

Theorem 3.6. Let $\sigma= \pm \infty$. For any $\lambda \in P^{+}$, the subspace $V(\lambda)_{\mathfrak{k} \sigma}$ of $\mathfrak{k}^{\sigma}$-fixed vectors in $V(\lambda)$ is at most one-dimensional. $V(\lambda)$ has non-zero $\mathfrak{k}^{\sigma}$-fixed vectors if and only if $\lambda=l\left(\varepsilon_{1}-\varepsilon_{n}\right)\left(l \in \mathbb{Z}_{+}\right)$. A $\mathfrak{k}^{\sigma}$-fixed vector in $V\left(\varepsilon_{1}-\varepsilon_{n}\right) \subset V^{*} \otimes V$ is given by $w_{\mathfrak{k} \sigma}=v_{n}^{*} \otimes v_{n}(\sigma=\infty)$ or $w_{\mathfrak{k} \sigma}=v_{1}^{*} \otimes v_{1}(\sigma=-\infty)$. The same statements hold with $\mathfrak{k}^{\sigma}$ replaced by $\left(\mathfrak{k}^{*}\right)^{\sigma}$. 
Proof. It is completely straightforward to check that $w_{\mathfrak{k} \infty}$ is indeed $\mathfrak{k}^{\infty}$-fixed. All the other statements concerning $\mathfrak{k}^{\infty}$-fixed vectors are proved in [NYM, Prop. 4.2].

Remark 3.7. Note that the expressions $w_{\mathfrak{k} \infty}=v_{n}^{*} \otimes v_{n}$ and $w_{\mathfrak{k} \sigma}=v_{1}^{*} \otimes v_{1}$ are special cases (up to a scalar multiple) of (3.10) ( $c=1, d=0$ and $c=0, d=1$ respectively). In other words, the expressions for $\mathfrak{k}^{\sigma}$-fixed and $\left(\mathfrak{k}^{*}\right)^{\sigma}$-fixed vectors in (3.10) are valid for any $c, d \geq 0,(c, d) \neq(0,0)$.

Specializing the notion of $\mathcal{U}_{q^{-}}$-invariant vectors in a $\mathcal{U}_{q}$-module $W$ to the $\mathcal{U}_{q^{-}}$ action on $\mathcal{A}_{q}$, we get the following terminology. An element $a \in \mathcal{A}_{q}$ is left resp. right invariant with respect to $u \in \mathcal{U}_{q}$ if $u \cdot a=\varepsilon(u) a$ resp. $a \cdot u=\varepsilon(u) a$ (cf. (3.3)).

For any $-\infty \leq \sigma \leq \infty$ let us define

$$
\mathcal{B}_{q}^{\sigma}=\mathcal{B}_{q}^{(c, d)}:=\left\{a \in \mathcal{A}_{q} \mid a \cdot \mathfrak{k}^{\sigma}=0\right\} .
$$

Then $\mathcal{B}_{q}^{\sigma}$ is a $*$-subalgebra and right coideal in $\mathcal{A}_{q}$, as follows from Proposition 3.1 (cf. [DK1, Prop. 1.9]). It is also invariant under the left action of $\mathcal{U}_{q}$ on $\mathcal{A}_{q}$.

Recall that $W(\lambda)\left(\lambda \in P^{+}\right)$denotes the subspace of $\mathcal{A}_{q}$ spanned by the coefficients of the (co-)representation $V(\lambda)$.

Theorem 3.8. Let $-\infty \leq \sigma \leq \infty$ be arbitrary. The irreducible decomposition of $\mathcal{B}_{q}^{\sigma}$ as a right $\mathcal{A}_{q}$-comodule resp. left $\mathcal{U}_{q}$-module is given by

$$
\mathcal{B}_{q}^{\sigma}=\bigoplus_{l \in \mathbb{Z}_{+}} V\left(l\left(\varepsilon_{1}-\varepsilon_{n}\right)\right),
$$

where the isotypical subspace of type $V\left(l\left(\varepsilon_{1}-\varepsilon_{n}\right)\right)$ is equal to the intersection of $\mathcal{B}_{q}^{\sigma}$ and $W(\lambda)$.

Proof. This follows from Corollary 3.3, Theorem 3.4, and Theorem 3.6. We omit the details, since the proof is completely analogous to the one given in [N, Prop. 4.1].

Our next goal will be to give a more explicit description of the subalgebra $\mathcal{B}_{q}^{\sigma} \subset$ $\mathcal{A}_{q}$. For this we need some results from [NYM, §1.5] on the linear independence of products of the $t_{i j}$. Let us remark that the commutation relations $R T_{1} T_{2}=T_{2} T_{1} R$ between the $t_{i j}$ are equivalent to the following relations:

$$
\begin{aligned}
& t_{i j} t_{i k}=q t_{i k} t_{i j}, \quad t_{l j} t_{i j}=q^{-1} t_{i j} t_{l j}, \quad t_{l k} t_{l j}=q^{-1} t_{l j} t_{l k}, \quad t_{l k} t_{i k}=q^{-1} t_{i k} t_{l k}, \\
& t_{l j} t_{i k}=t_{i k} t_{l j}, \quad t_{l k} t_{i j}=t_{i j} t_{l k}-\left(q-q^{-1}\right) t_{i k} t_{l j} \quad(j<k, i<l) .
\end{aligned}
$$

Using this explicit form of the relations, one can construct a basis of monomials in the $t_{i j}$ for the subalgebra of $\mathcal{A}_{q}$ generated by the $t_{i j}$. Let $M(n, \mathbb{N})$ denote the space of $n \times n$ matrices with non-negative integer coefficients. For any matrix $A=\left(a_{i j}\right) \in M(n, \mathbb{N})$, define a monomial $t^{A}:=t_{11}^{a_{11}} t_{12}^{a_{12}} \cdots t_{21}^{a_{21}} t_{22}^{a_{22}} \cdots t_{n n}^{a_{n n}}$, where the factors are arranged according to the lexicographical order on the indices $(i, j)$. The following theorem is proved in [NYM, Thm. 1.4]:

Theorem 3.9. The elements $t^{A} \in \mathcal{A}_{q}(A \in M(n, \mathbb{N}))$ form a linear basis of the subalgebra $M_{q} \subset \mathcal{A}_{q}$ generated by the $t_{i j}(1 \leq i, j \leq n)$. 
With any $A=\left(a_{i j}\right) \in M(n, \mathbb{N})$ we associate a sequence of integers $r(A)$ given by

$$
r(A):=\left(\sum_{i j} a_{i j}, a_{11}, a_{12}, \ldots, a_{21}, a_{22}, \ldots, a_{n n}\right) \in \mathbb{N}^{n^{2}+1} .
$$

The lexicographic ordering on $\mathbb{N}^{n^{2}+1}$ then induces a total ordering $\preceq$ on $M(n, \mathbb{N})$ in the obvious way. Note that this ordering is compatible with the additive structure on $M(n, \mathbb{N})$. It now follows that any non-zero element $\varphi \in M_{q}$ can be uniquely written as a linear combination of the form

$$
\varphi=c_{A} t^{A}+\sum_{B \prec A} c_{B} t^{B}
$$

where $c_{A} \neq 0$. Let us call $A=: d(\varphi)$ the degree of $\varphi$. Suppose $\varphi=t_{i_{1} j_{1}} \cdots t_{i_{p} j_{p}}$ is any monomial in the $t_{i j}$. It then follows from the relations (3.17) and Theorem 3.9 that $d(\varphi)=A$, where the coefficient $a_{i j}$ of $A=\left(a_{i j}\right)$ is defined as the number of occurrences of $t_{i j}$ in the product $t_{i_{1} j_{1}} \cdots t_{i_{p} j_{p}}$. It is now easy to see that a product of non-zero elements in $M_{q}$ is again non-zero and that

$$
d(\varphi \psi)=d(\varphi)+d(\psi), \quad 0 \neq \varphi, \psi \in M_{q} .
$$

Since every element in $\mathcal{A}_{q}$ can be written as a product of a power of $\operatorname{det}_{q}^{-1}$ and an element in $M_{q}$ (recall that $\operatorname{det}_{q}^{-1}$ is central in $\mathcal{A}_{q}$ ), we get:

Lemma 3.10. The algebra $A_{q}$ has no (left or right) zero divisors.

Corresponding to the contragredient representation $V^{*}$ we have a right $\mathcal{U}_{q^{-}}$ module $\operatorname{Hom}\left(V^{*}, \mathbb{C}\right)$, whose underlying vector space can be naturally identified with $V$. The assignment $v_{i} \mapsto q^{\left\langle 2 \rho, \varepsilon_{i}\right\rangle} v_{i}^{*}$ (cf. (3.7)) defines an intertwining operator from $\operatorname{Hom}\left(V^{*}, \mathbb{C}\right)$ into $\left(V^{*}\right)^{\circ}$. The mapping

$$
V^{*} \otimes \operatorname{Hom}\left(V^{*}, \mathbb{C}\right) \rightarrow \mathcal{A}_{q}, \quad v_{i}^{*} \otimes v_{j} \mapsto t_{j i}^{*}
$$

is an injective $\mathcal{U}_{q}$-bimodule homomorphism.

Proposition 3.11. Let $-\infty \leq \sigma \leq \infty$ be arbitrary. We put

$$
x_{i j}:=d t_{1 i}^{*} t_{1 j}+c t_{n i}^{*} t_{n j}+\sqrt{c d} t_{n i}^{*} t_{1 j}+\sqrt{c d} t_{1 i}^{*} t_{n j} \in \mathcal{A}_{q} \quad(1 \leq i, j \leq n) .
$$

The subspace spanned by the $x_{i j}(1 \leq i, j \leq n)$ is invariant under the left $\mathcal{U}_{q}$-action. The linear mapping defined by

$$
v_{i}^{*} \otimes v_{j} \mapsto x_{i j}: V^{*} \otimes V \rightarrow \mathcal{A}_{q}
$$

is an injective operator intertwining the left $\mathcal{U}_{q}$-actions. The $x_{i j}$ are right $\mathfrak{k}^{\sigma}$ invariant and satisfy $x_{i j}^{*}=x_{j i}$. They generate the subalgebra $\mathcal{B}_{q}^{\sigma}$. A highest weight vector for $V\left(l\left(\varepsilon_{1}-\varepsilon_{n}\right)\right) \subset \mathcal{B}_{q}^{\sigma}$ is $x_{n 1}^{l}$.

Proof. It follows from (3.20) and the remarks preceding this proposition, that the operator (3.21) intertwines the left actions of $\mathcal{U}_{q}$ on $V^{*} \otimes V$ and $\mathcal{A}_{q}$. Hence, the subspace spanned by the $x_{i j}$ is invariant. It is proved in [NYM, Corollary to Prop. 1.1] that $\sum_{k} q^{2(i-k)} t_{i k}^{*} t_{j k}=\delta_{i j} 1 \in \mathcal{A}_{q}(1 \leq i, j \leq n)$. It follows that $\sum_{k} q^{2(n-k)} x_{k k}=c+q^{2(n-1)} d \in \mathcal{A}_{q}$. Since $c+q^{2(n-1)} d \neq 0$, the trivial representation occurs with non-zero multiplicity in the irreducible decomposition of the subspace spanned by the $x_{i j}$. On the other hand, the element $x_{n 1} \in \mathcal{A}_{q}$ has weight $\varepsilon_{1}-\varepsilon_{n}$ and is annihilated by the $L_{i j}^{-}(i>j)$, being the image of the highest weight vector $v_{n}^{*} \otimes v_{1}$. If we can prove that $x_{n 1}$ is non-zero, it will follow from (3.8) that the operator (3.21) is injective. If $\sigma$ is finite, $x_{n 1}$ is clearly non-zero, since $\varepsilon\left(x_{n 1}\right)=\sqrt{c d} \neq 0$. If $\sigma=\infty$ 
it follows from Lemma 3.10 that $x_{n 1}=t_{n n}^{*} t_{n 1}$ is non-zero. Next, the fact that the $x_{i j}$ are right $\mathfrak{k}^{\sigma}$-invariant follows immediately from the expression for the $\left(\mathfrak{k}^{*}\right)^{\sigma}$-fixed vector in (3.10) and (1.12), (3.19). The property $x_{i j}^{*}=x_{j i}$ is completely trivial. It therefore remains to prove that the $x_{i j}$ generate the whole subalgebra $\mathcal{B}_{q}^{\sigma}$. Indeed, a second application of Lemma 3.10 shows that $x_{n 1}^{l}$ is non-zero. But then it is a highest weight vector of weight $l\left(\varepsilon_{1}-\varepsilon_{n}\right)$. Hence, in the subalgebra generated by the $x_{i j}$, the isotypical subspace of type $V\left(l\left(\varepsilon_{1}-\varepsilon_{n}\right)\right)$ is non-zero. The assertion now follows from Theorem 3.8.

Remark 3.12. Instead of $\mathcal{B}_{q}^{\sigma}$, one can also consider the subalgebra $\mathcal{C}_{q}^{\sigma}$ of left $\mathfrak{k}^{\sigma}-$ invariant functions. It is a right $\mathcal{U}_{q}$-module and decomposes as

$$
\mathcal{C}_{q}^{\sigma}=\bigoplus_{l \in \mathbb{Z}_{+}} V\left(l\left(\varepsilon_{1}-\varepsilon_{n}\right)\right)^{\circ} .
$$

The elements $y_{i j}:=q d t_{i 1}^{*} t_{j 1}+q^{-1} c t_{i n}^{*} t_{j n}+\sqrt{c d} t_{i n}^{*} t_{j 1}+\sqrt{c d} t_{i 1}^{*} t_{j n} \in \mathcal{A}_{q}(1 \leq i, j \leq$ $n$ ) are left $\mathfrak{k}^{\sigma}$-invariant and generate the algebra $\mathcal{C}_{q}^{\sigma}$. A highest weight vector in $V\left(l\left(\varepsilon_{1}-\varepsilon_{n}\right)\right)^{\circ}$ is $y_{n 1}^{l}$. The assignment $v_{i}^{*} \otimes v_{j} \mapsto q^{-\left\langle 2 \rho, \varepsilon_{i}\right\rangle} y_{i j}$ defines an injective intertwining operator of $\left(V^{*} \otimes V\right)^{\circ}$ into $\mathcal{C}_{q}^{\sigma}$.

\section{A $q$-Analogue of the Hopf fibration}

In this section we shall describe a more geometrically inspired way to construct the algebra $\mathcal{B}_{q}^{\sigma}$ in a uniform way for all values of $\sigma$.

Let $\mathcal{A}_{q}(\tilde{S})=\mathcal{A}_{q}\left(\tilde{S}^{2 n-1}\right)$ denote the algebra generated by the symbols $z_{i}, w_{i}$ $(1 \leq i \leq n)$ and $c, d$ subject to the relations

$$
\begin{aligned}
& \text { (i) } z_{i} z_{j}=q z_{j} z_{i}, q w_{i} w_{j}=w_{j} w_{i} \quad(1 \leq i<j \leq n), \\
& \text { (ii) } w_{j} z_{i}=q z_{i} w_{j} \quad(1 \leq i \neq j \leq n), \\
& \text { (iii) } w_{j} z_{j}=z_{j} w_{j}+\left(1-q^{2}\right) \sum_{1 \leq k<j} z_{k} w_{k}-\left(1-q^{2}\right) q^{-2} d \quad(1 \leq j \leq n), \\
& \text { (iv) } \sum_{k=1}^{n} z_{k} w_{k}=c+q^{-2} d .
\end{aligned}
$$

As can be easily checked, these defining relations imply the following relations $(1 \leq j \leq n)$ :

$$
\begin{aligned}
& \text { (v) } c z_{j}=z_{j} c, c w_{j}=w_{j} c, c d=d c \\
& \text { (vi) } q^{-2} d z_{j}=z_{j} d, d w_{j}=q^{-2} w_{j} d, \\
& \text { (vii) } z_{j} w_{j}=w_{j} z_{j}+\left(1-q^{-2)} \sum_{1 \leq k<j} q^{2(j-k)} w_{k} z_{k}-\left(1-q^{-2}\right) q^{2(j-1)} d,\right. \\
& \text { (viii) } \sum_{k=1}^{n} q^{2(n-k)} w_{k} z_{k}=c+q^{2(n-1)} d .
\end{aligned}
$$

Note that $c$ is central, but $d$ is not. The algebra $\mathcal{A}_{q}\left(\tilde{S}^{2 n-1}\right)$ can be considered as the algebra of functions on the total space of a family of quantum $(2 n-1)$-spheres. The case $n=2$ was considered in [NM3]. The algebra $\mathcal{A}_{q}\left(\tilde{S}^{2 n-1}\right)$ was introduced in general in $[\mathrm{KV}]$. 
There is a unique $*$-structure on the algebra $\mathcal{A}_{q}\left(\tilde{S}^{2 n-1}\right)$ such that

$$
z_{j}^{*}=w_{j}, \quad c^{*}=c, \quad d^{*}=d .
$$

Let us define algebra homomorphisms

$$
R: \mathcal{A}_{q}(\tilde{S}) \rightarrow \mathcal{A}_{q}(\tilde{S}) \otimes \mathcal{A}_{q}, \quad L: \mathcal{A}_{q}(\tilde{S}) \rightarrow \mathcal{A}(U(1)) \otimes \mathcal{A}_{q}(\tilde{S})
$$

by putting $(1 \leq j \leq n)$

$$
\begin{aligned}
& R\left(z_{j}\right)=\sum_{i=1}^{n} z_{i} \otimes t_{i j}, R\left(w_{j}\right)=\sum_{i=1}^{n} w_{i} \otimes S\left(t_{j i}\right), R(c)=c \otimes 1, R(d)=d \otimes 1 \\
& L\left(z_{j}\right)=z \otimes z_{j}, L\left(w_{j}\right)=z^{-1} \otimes w_{j}, L(c)=1 \otimes c, L(d)=1 \otimes d
\end{aligned}
$$

In this way, $\mathcal{A}_{q}(\tilde{S})$ becomes a two-sided $\left(\mathcal{A}(U(1)), \mathcal{A}_{q}\right)$-comodule algebra. Note that $R$ and $L$ commute with the *-operations. Let us remark that by "differentiating" the right $\mathcal{A}_{q}$-coaction one obtains a left $\mathcal{U}_{q}$-module structure on $\mathcal{A}_{q}(\tilde{S})$.

The algebra $\mathcal{A}_{q}(\tilde{S})$ can be realized inside a suitable extension of $\mathcal{A}_{q}$ by a $q$-shift operator. Let $\mathcal{C}:=\mathbb{C}[\alpha, \beta, \gamma, \delta]$ denote the free polynomial algebra in the commuting variables $\alpha, \beta, \gamma, \delta$. Define an algebra automorphism $\theta: \mathcal{C} \rightarrow \mathcal{C}$ by

$$
\theta(\alpha)=\alpha, \quad \theta(\beta)=q \beta, \quad \theta(\gamma)=q \gamma, \quad \theta(\delta)=\delta .
$$

Let $\mathcal{C}\left[\theta^{ \pm 1}\right]$ denote the subalgebra of $\operatorname{End}_{\mathbb{C}}(\mathcal{C})$ generated by left multiplication by $\alpha, \beta, \gamma, \delta$ and the $q$-shift operators $\theta, \theta^{-1}$. One has the obvious algebra isomorphism $\mathcal{C}\left[\theta^{ \pm 1}\right] \cong \mathbb{C}\left[\theta^{ \pm 1}\right] \otimes \mathbb{C}[\alpha, \beta, \gamma, \delta]$, where the (twisted) multiplication on the tensor product on the right-hand side is defined by the rule $\theta P=\theta(P) \theta(P \in \mathbb{C}[\alpha, \beta, \gamma, \delta])$. There is a unique $*$-structure on $\mathcal{C}\left[\theta^{ \pm 1}\right]$ such that

$$
\theta^{*}=\theta^{-1}, \quad \alpha^{*}=\delta, \quad \beta^{*}=-\gamma .
$$

Now consider the $*$-algebra $\mathcal{C}\left[\theta^{ \pm 1}\right] \otimes \mathcal{A}_{q}$, the multiplication and the $*$-structure on the tensor product being defined as usual. We define a two-sided $\left(\mathcal{A}(U(1)), \mathcal{A}_{q}\right)$ comodule structure on $\mathcal{C}\left[\theta^{ \pm 1}\right] \otimes \mathcal{A}_{q}$ with structure mappings

$$
\begin{gathered}
R: \mathcal{C}\left[\theta^{ \pm 1}\right] \otimes \mathcal{A}_{q} \rightarrow \mathcal{C}\left[\theta^{ \pm 1}\right] \otimes \mathcal{A}_{q} \otimes \mathcal{A}_{q}, \quad R:=\mathrm{id} \otimes \Delta, \\
L: \mathcal{C}\left[\theta^{ \pm 1}\right] \otimes \mathcal{A}_{q} \rightarrow \mathcal{A}(U(1)) \otimes \mathcal{C}\left[\theta^{ \pm 1}\right] \otimes \mathcal{A}_{q}, \quad L(\theta):=z^{-1} \otimes \theta,
\end{gathered}
$$

$L$ acting trivially on all the other generators.

Theorem 4.1. There is a unique algebra homomorphism $\Phi: \mathcal{A}_{q}(\tilde{S}) \rightarrow \mathcal{C}\left[\theta^{ \pm 1}\right] \otimes \mathcal{A}_{q}$ such that $(1 \leq j \leq n)$

$$
\Phi\left(z_{j}\right)=\theta^{-1}\left(\gamma t_{1 j}+\delta t_{n j}\right), \Phi\left(w_{j}\right)=\left(-\beta t_{1 j}^{*}+\alpha t_{n j}^{*}\right) \theta, \Phi(c)=\alpha \delta, \Phi(d)=-\beta \gamma .
$$

The mapping $\Phi$ is injective, commutes with the *-structures, and intertwines the two-sided $\left(\mathcal{A}(U(1)), \mathcal{A}_{q}\right)$-comodule structures.

Proof. All assertions are obvious except the existence and the injectivity. To check the existence we need some information about the commutation relations between the $t_{i j}$ and the $t_{i j}^{*}$ in $\mathcal{A}_{q}$. Put $\check{R}=P R$ with $P: V \otimes V \rightarrow V \otimes V$ the permutation operator. The commutation relations between the $t_{i j}$ can be rewritten as $\check{R} T_{1} T_{2}=T_{1} T_{2} \check{R}$. Using $T S(T)=S(T) T=I$, one deduces the identity 
$S(T)_{1} \check{R} T_{1}=T_{2} \check{R} S(T)_{2}$. Making this explicit, one gets $(1 \leq i, j, k, l \leq n)$

$$
q^{\delta_{j l}} t_{l i}^{*} t_{j k}+\left(q-q^{-1}\right) \delta_{j l} \sum_{m<j} t_{m i}^{*} t_{m k}=q^{\delta_{i k}} t_{j k} t_{l i}^{*}+\left(q-q^{-1}\right) \delta_{i k} \sum_{m>i} t_{j m} t_{l m}^{*} .
$$

Using these relations it is straightforward to check that $\Phi$ is well-defined. To prove that $\Phi$ is injective, first observe that the algebra $\mathcal{A}_{q}(\tilde{S})$ has a natural grading preserved by the $\mathcal{U}_{q}$-action such that $\operatorname{deg}\left(z_{i}\right)=\operatorname{deg}\left(w_{i}\right)=1(1 \leq i \leq n)$ and $\operatorname{deg}(c)=\operatorname{deg}(d)=2$. Suppose the $\Phi\left(w_{n}^{m} z_{1}^{l}\right)$ are non-zero. Then they are highest weight vectors of weight $l \varepsilon_{1}-m \varepsilon_{n}$ with respect to the $\mathcal{U}_{q}$-action, and so are the $w_{n}^{m} z_{1}^{l} \in \mathcal{A}_{q}(\tilde{S})$. Let us denote the $\mathcal{U}_{q}$-submodule generated by $w_{n}^{m} z_{1}^{l}(l, m$ fixed) by $V\left(l \varepsilon_{1}-m \varepsilon_{n}\right)$. The elements $w_{n}^{m} z_{1}^{l} c^{r} d^{s}$ (l,m fixed, $r, s \geq 0$ arbitrary) are linearly independent in $\mathcal{A}_{q}(\tilde{S})$, since their images under $\Phi$ are in $\mathcal{C}\left[\theta^{ \pm 1}\right] \otimes \mathcal{A}_{q}$. This shows that multiplication defines an injective linear mapping

$$
\bigoplus_{l, m \geq 0} V\left(l \varepsilon_{1}-m \varepsilon_{n}\right) \otimes \mathbb{C}[c, d] \hookrightarrow \mathcal{A}_{q}(\tilde{S})
$$

intertwining the $\mathcal{U}_{q}$-actions. On the other hand, by (4.1) multiplication defines a surjective graded linear mapping

$$
\mathbb{C}_{q}\left[w_{1}, \ldots, w_{n}\right] \otimes \mathbb{C}_{q}\left[z_{1}, \ldots, z_{n}\right] \otimes \mathbb{C}[d] \rightarrow \mathcal{A}_{q}(\tilde{S})
$$

intertwining the $\mathcal{U}_{q}$-actions. Here the algebras on the left-hand side are the wellknown polynomial algebras in $q$-commuting variables (cf. (4.1)) with the natural $\mathcal{U}_{q}$-symmetry and grading. Their respective irreducible decompositions are given by $\mathbb{C}_{q}\left[z_{1}, \ldots, z_{n}\right]=\bigoplus_{l \geq 0} V\left(l \varepsilon_{1}\right)$ and $\mathbb{C}_{q}\left[w_{1}, \ldots, w_{n}\right]=\bigoplus_{m \geq 0} V\left(-m \varepsilon_{n}\right)$. For any $N \geq 0$, the mappings (4.8) and (4.9) place lower and upper bounds respectively on the dimension of the homogeneous component in $\mathcal{A}_{q}(\tilde{S})$ of degree $N$. An easy counting argument using the well-known fact that the tensor product $V\left(l \varepsilon_{1}\right) \otimes V\left(-m \varepsilon_{n}\right)$ decomposes as the direct sum $\bigoplus_{i=0}^{l \wedge m} V\left((l-i) \varepsilon_{1}-(m-i) \varepsilon_{n}\right)$ shows that these upper and lower bounds are actually equal. This implies that the mapping (4.8) is surjective. Obviously, the restriction of $\Phi$ to each of the components $V\left(l \varepsilon_{1}-m \varepsilon_{n}\right) \otimes \mathbb{C}[c, d]$ in (4.8) is injective, the $\mathcal{U}_{q}$-modules $V\left(l \varepsilon_{1}-m \varepsilon_{n}\right)$ being irreducible. The injectivity of $\Phi$ therefore follows from the following lemma.

Lemma 4.2. The elements

$$
\Phi\left(w_{n}^{m} z_{1}^{l}\right)=\left\{\left(-\beta t_{1 n}^{*}+\alpha t_{n n}^{*}\right) \theta\right\}^{m}\left\{\theta^{-1}\left(\gamma t_{11}+\delta t_{n 1}\right)\right\}^{l} \quad(l, m \geq 0)
$$

in the algebra $\mathcal{C}\left[\theta^{ \pm 1}\right] \otimes \mathcal{A}_{q}$ are non-zero.

Proof. We are going to apply Theorem 3.9, but for this we need a more explicit description of the $*$-operation. For any $1 \leq i \leq n$ denote by $\left(i_{1}, \ldots, i_{n-1}\right)$ the sequence of length $n-1$ obtained from $(1, \ldots, n)$ by deleting $i$. Define the quantum principal minor $\xi_{j}^{i} \in \mathcal{A}_{q}$ by

$$
\xi_{j}^{i}:=\sum_{w \in \mathfrak{S}_{n-1}}(-q)^{l(w)} t_{i_{w(1)} j_{1}} \cdots t_{i_{w(n-1)} j_{n-1}} \quad(1 \leq i, j \leq n) .
$$

Here $\mathfrak{S}_{n-1}$ is the permutation group on $n-1$ letters, and $l(w)$ denotes the length of $w$. It is well-known (cf. [RTF, Thm. 4], [NYM, (1.24)]) that the $*$-operation is given on the generators $t_{i j}(1 \leq i, j \leq n)$ by

$$
t_{i j}^{*}=(-q)^{j-i} \operatorname{det}_{q}^{-1} \xi_{j}^{i} \quad(1 \leq i, j \leq n) .
$$


Recall the notion of degree for a monomial in the $t_{i j}$ (cf. section 3). On the one hand, $d\left(t_{n 1}\right) \prec d\left(t_{11}\right)$. On the other hand, it is easy to see from (4.10) that $d\left(\xi_{n}^{1}\right) \prec d\left(\xi_{n}^{n}\right)$. Let us put $A:=d\left(t_{11}\right)$ and $B:=d\left(\xi_{n}^{n}\right)$. Since $t_{11} t_{n 1}=q t_{n 1} t_{11}$ and $t_{n n}^{*} t_{1 n}^{*}=q t_{1 n}^{*} t_{n n}^{*}$, we can expand $\Phi\left(w_{n}^{m} z_{1}^{l}\right)$ as

$$
\sum_{M=\left(m_{1}, m_{2}, l_{1}, l_{2}\right)} c_{M} P_{M}(\theta, \alpha, \beta, \gamma, \delta) \otimes \operatorname{det}_{q}^{-m_{1}-m_{2}}\left(\xi_{n}^{1}\right)^{m_{1}}\left(\xi_{n}^{n}\right)^{m_{2}} t_{11}^{l_{1}} t_{n 1}^{l_{2}},
$$

where the sum runs over all quadruples $M=\left(m_{1}, m_{2}, l_{1}, l_{2}\right)$ of non-negative integers such that $m_{1}+m_{2}=m$ and $l_{1}+l_{2}=l$, the $c_{M}$ are non-zero constants, and the $P_{M}(\theta, \alpha, \beta, \gamma, \delta)$ are certain (commutative) monomials in the variables $\theta, \alpha, \beta, \gamma, \delta$. A moment's consideration shows that for all $\left(m_{1}, m_{2}, l_{1}, l_{2}\right)$

$$
d\left(\left(\xi_{n}^{1}\right)^{m_{1}}\left(\xi_{n}^{n}\right)^{m_{2}} t_{11}^{l_{1}} t_{n 1}^{l_{2}}\right) \preceq m B+l A,
$$

and that equality holds if and only if $\left(m_{1}, m_{2}\right)=(0, m)$ and $\left(l_{1}, l_{2}\right)=(l, 0)$. The corresponding coefficient $c_{(0, m, l, 0)}$ equals $q^{k} \alpha^{m} \gamma^{l}$ for a certain $k \in \mathbb{Z}$. This proves that $w_{n}^{m} z_{1}^{l}$ is non-zero.

As a corollary of the proof of Theorem 4.1 we have

Corollary 4.3. The isotypical decomposition of $\mathcal{A}_{q}(\tilde{S})$ with respect to the $\mathcal{A}_{q^{-}}$ coaction is

$$
\mathcal{A}_{q}(\tilde{S}) \cong \bigoplus_{l, m \geq 0} V\left(l \varepsilon_{1}-m \varepsilon_{n}\right) \otimes \mathbb{C}[c, d] .
$$

Here $V\left(l \varepsilon_{1}-m \varepsilon_{n}\right)$ is the $\mathcal{A}_{q}$-subcomodule of $\mathcal{A}_{q}(\tilde{S})$ generated by the highest weight vector $w_{n}^{m} z_{1}^{l}$. The isomorphism $\cong$ is given (from right to left) by multiplication.

Let us now consider $U(1)$-invariance in $\mathcal{A}_{q}(\tilde{S})$. Recall that in the classical case dividing out the sphere $S^{2 n-1} \subset \mathbb{C}^{n}$ by its natural $U(1)$-action yields the complex projective space $\mathbb{C P}^{n-1}$ (Hopf fibration). We define

$$
\mathcal{A}_{q}\left(\widetilde{\mathbb{C P}}^{n-1}\right):=\left\{a \in \mathcal{A}_{q}\left(\tilde{S}^{2 n-1}\right) \mid L(a)=1 \otimes a\right\} .
$$

Clearly, $\mathcal{A}_{q}\left(\widetilde{\mathbb{C P}^{n-1}}\right)$ is a $*$-subalgebra and $\mathcal{A}_{q}$-subcomodule of $\mathcal{A}_{q}(\tilde{S})$. It follows immediately from Corollary 4.3 that $\mathcal{A}_{q}\left(\widetilde{\mathbb{C P}}^{n-1}\right)$ is generated by the elements $\tilde{x}_{i j}:=$ $w_{i} z_{j} \in \mathcal{A}_{q}(\tilde{S})$ and $c, d$. Note that both $c$ and $d$ are central in $\mathcal{A}_{q}\left(\widetilde{\mathbb{C P}^{n-1}}\right)$. Let $\mathcal{A}_{q}\left(\mathbb{C P}_{q}^{n-1}(c, d)\right)$ denote the algebra obtained from $\mathcal{A}_{q}\left(\widetilde{\mathbb{C P}^{n-1}}\right)$ by specialization of $c, d$ to real non-negative values not both equal to zero. It has a natural right $\mathcal{A}_{q}$-comodule structure with an irreducible decomposition given by

$$
\mathcal{A}_{q}\left(\mathbb{C P}_{q}^{n-1}(c, d)\right)=\bigoplus_{l \geq 0} V\left(l\left(\varepsilon_{1}-\varepsilon_{n}\right)\right) .
$$

The algebras $\mathcal{A}_{q}\left(\widetilde{\mathbb{C P}}^{n-1}\right)$ and its specialization already appeared in $[\mathrm{KV}]$. For $n=2$, the algebras $\mathcal{A}_{q}\left(\mathbb{C P}^{n-1}(c, d)\right)(c, d \geq 0)$ coincide with the algebras of functions on the quantum spheres introduced in $[\mathrm{P}]$.

Theorem 4.4. Let $c_{0}, d_{0} \geq 0$ be real numbers not both equal to zero. There is a unique algebra homomorphism

$$
\mathcal{A}_{q}\left(\widetilde{\mathbb{C P}}^{n-1}\right) \rightarrow \mathcal{B}_{q}^{\left(c_{0}, d_{0}\right)}, \quad \tilde{x}_{i j} \mapsto x_{i j}, c \mapsto c_{0}, d \mapsto d_{0} .
$$

This mapping induces a *-algebra isomorphism of $\mathcal{A}_{q}\left(\mathbb{C P}_{q}^{n-1}\left(c_{0}, d_{0}\right)\right)$ onto $\mathcal{B}_{q}^{\left(c_{0}, d_{0}\right)}$ intertwining the $\mathcal{A}_{q}$-coactions. 
Proof. Define an algebra homomorphism $\varphi: \mathbb{C}[\alpha, \beta, \gamma, \delta] \rightarrow \mathbb{C}$ by the rule

$$
\varphi(\alpha)=\varphi(\delta)=\sqrt{c_{0}}, \quad \varphi(\beta)=-\sqrt{d_{0}}, \quad \varphi(\gamma)=\sqrt{d_{0}} .
$$

Obviously, $\varphi$ maps $c, d \in \mathbb{C}[\alpha, \beta, \gamma, \delta]$ onto $c_{0}, d_{0} \in \mathbb{C}$. Since $\Phi\left(\mathcal{A}_{q}\left(\widetilde{\mathbb{C P}^{n-1}}\right)\right)$ is contained in the subspace $\mathbb{C}[\alpha, \beta, \gamma, \delta] \otimes \mathcal{A}_{q}$ of $\mathcal{C}\left[\theta^{ \pm 1}\right] \otimes \mathcal{A}_{q}$, the composition ( $\varphi \otimes$ id) $\circ \Phi$ is well-defined on $\mathcal{A}_{q}\left(\widetilde{\mathbb{C P}}^{n-1}\right)$. It is a $*$-algebra homomorphism into $\mathcal{A}_{q}$ mapping $\tilde{x}_{i j}=w_{i} z_{j}$ onto

$$
\begin{aligned}
\left(-\varphi(\beta) t_{1 i}^{*}\right. & \left.+\varphi(\alpha) t_{n i}^{*}\right)\left(\varphi(\gamma) t_{1 j}+\varphi(\delta) t_{n j}\right) \\
& =d_{0} t_{1 i}^{*} t_{1 j}+c_{0} t_{n i}^{*} t_{n j}+\sqrt{c_{0} d_{0}} t_{n i}^{*} t_{1 j}+\sqrt{c_{0} d_{0}} t_{1 i}^{*} t_{n j} .
\end{aligned}
$$

This proves the existence of (4.14). The remaining assertions now follow trivially from (4.13), Theorem 3.8, Prop. 3.11 and the definitions.

We shall call either $\mathcal{A}_{q}\left(\mathbb{C P}^{n-1}(c, d)\right)$ or $\mathcal{B}_{q}^{(c, d)}$ the algebra of representative functions on the quantum projective space $\mathbb{C P}_{q}^{n-1}(c, d)$.

Corollary 4.5. There exists a unique *-algebra homomorphism

$$
\tilde{\varepsilon}: \mathcal{A}_{q}\left(\mathbb{C P}^{n-1}(c, d)\right) \rightarrow \mathbb{C}
$$

such that $\tilde{\varepsilon}\left(x_{11}\right)=d, \tilde{\varepsilon}\left(x_{n n}\right)=c, \tilde{\varepsilon}\left(x_{1 n}\right)=\sqrt{c d}, \tilde{\varepsilon}\left(x_{n 1}\right)=\sqrt{c d}$, and $\tilde{\varepsilon}\left(x_{i j}\right)=0$ in all other cases.

Proof. Simply take $\tilde{\varepsilon}$ equal to the composition of the isomorphism described in Theorem 4.4 and the counit $\varepsilon: \mathcal{A}_{q} \rightarrow \mathbb{C}$.

The $*$-homomorphism $\tilde{\varepsilon}$ can be regarded as a "classical" point in the quantum projective space $\mathbb{C P}_{q}^{n-1}(c, d)$ (cf. [DK1, Prop. 1.1]).

\section{ZONAL SPHERICAL FUNCTIONS}

Let us fix parameters $-\infty \leq \sigma, \tau \leq \infty$. We define the $*$-subalgebra of $(\sigma, \tau)$-biinvariant functions in $\mathcal{A}_{q}$ as

$$
\mathcal{H}^{(\sigma, \tau)}:=\left\{a \in \mathcal{A}_{q} \mid \mathfrak{k}^{\sigma} \cdot a=0 \quad \text { and } \quad a \cdot \mathfrak{k}^{\tau}=0\right\},
$$

An element $a \in \mathcal{A}_{q}$ is called $(\sigma, \tau)$-spherical if it is $(\sigma, \tau)$-bi-invariant and contained in $W(\lambda)$ for some $\lambda \in P^{+}$.

Proposition 5.1. If we put $\mathcal{H}^{(\sigma, \tau)}(\lambda):=\mathcal{H}^{(\sigma, \tau)} \cap W(\lambda)$, then

$$
\mathcal{H}^{(\sigma, \tau)}=\bigoplus_{l \in \mathbb{Z}_{+}} \mathcal{H}^{(\sigma, \tau)}\left(l\left(\varepsilon_{1}-\varepsilon_{n}\right)\right),
$$

and each of the spaces $\mathcal{H}^{(\sigma, \tau)}\left(l\left(\varepsilon_{1}-\varepsilon_{n}\right)\right)$ is one-dimensional.

Proof. This is a direct consequence of Theorems 3.8, 3.6, 3.4, and Corollary 3.3.

Let us now suppose that $\sigma, \tau$ are finite. It follows immediately from (3.10) and Proposition 3.11 that the direct sum $\mathcal{H}^{(\sigma, \tau)}(0) \oplus \mathcal{H}^{(\sigma, \tau)}\left(\varepsilon_{1}-\varepsilon_{n}\right)$ is spanned by the unit element $1 \in \mathcal{A}_{q}$ and the element

$$
x^{(\sigma, \tau)}:=\frac{1}{2}\left(\hat{x}_{1 n}+\hat{x}_{n 1}+q^{\sigma+1} \hat{x}_{11}+q^{-\sigma-1} \hat{x}_{n n}-\left(q^{\sigma+\tau+1}+q^{-\sigma-\tau-1}\right)\right) \in \mathcal{A}_{q} .
$$


Here we take $\left(q^{\tau}=\sqrt{d / c}\right)$ :

$$
\hat{x}_{i j}:=q^{\tau} t_{1 i}^{*} t_{1 j}+q^{-\tau} t_{n i}^{*} t_{n j}+t_{n i}^{*} t_{1 j}+t_{1 i}^{*} t_{n j} \in \mathcal{A}_{q} \quad(1 \leq i, j \leq n) .
$$

Note that the $\hat{x}_{i j}$ differ from the $x_{i j}$ (cf. (3.20)) by a scalar multiple. One has $\left(x^{(\sigma, \tau)}\right)^{*}=x^{(\sigma, \tau)}$. The following lemma is obvious:

Lemma 5.2. Let $\sigma, \tau$ be finite. Under the restriction mapping ${ }_{\mid \mathbb{T}}: \mathcal{A}_{q} \longrightarrow \mathcal{A}(\mathbb{T})$ we have

$$
\hat{x}_{11} \mapsto q^{\tau}, \quad \hat{x}_{n n} \mapsto q^{-\tau}, \quad \hat{x}_{1 n} \mapsto z_{1}^{-1} z_{n}, \quad \hat{x}_{n 1} \mapsto z_{1} z_{n}^{-1},
$$

and all the other $x_{i j}$ are mapped onto 0 . In particular, the image of $x^{(\sigma, \tau)}$ equals $\frac{1}{2}\left(z_{1} z_{n}^{-1}+z_{1}^{-1} z_{n}\right)$.

Proposition 5.3. Let $\sigma, \tau$ be finite. The algebra $\mathcal{H}^{(\sigma, \tau)}$ is generated by $x^{(\sigma, \tau)}$ and hence commutative. If we put $z:=z_{1} z_{n}^{-1} \in \mathcal{A}(\mathbb{T})$ then the restriction of the mapping ${ }_{\mid \mathbb{T}}$ to $\mathcal{H}^{(\sigma, \tau)} \subset \mathcal{A}_{q}$ is an injective *-algebra homomorphism onto the polynomial algebra $\mathcal{H}_{\mid \mathbb{T}}^{(\sigma, \tau)}:=\mathbb{C}\left[\frac{1}{2}\left(z+z^{-1}\right)\right] \subset \mathcal{A}(\mathbb{T})$.

Proof. Recall that $\hat{x}_{n 1}^{l} \in \mathcal{A}_{q}\left(l \in \mathbb{Z}_{+}\right)$is a (non-zero) highest weight vector of weight $l\left(\varepsilon_{1}-\varepsilon_{n}\right)$ (cf. Proposition 3.11). Hence the component of $\left(x^{(\sigma, \tau)}\right)^{l}$ in $\mathcal{H}^{(\sigma, \tau)}\left(l\left(\varepsilon_{1}-\varepsilon_{n}\right)\right)$ is non-zero. This implies that $x^{(\sigma, \tau)}$ generates the algebra $\mathcal{H}^{(\sigma, \tau)}$. By Lemma 5.2 the restriction of $\left(x^{(\sigma, \tau)}\right)^{l}$ to $\mathbb{T}$ is equal to a non-zero scalar multiple of $z_{1}^{l} z_{n}^{-l}$ plus some lower order terms w.r.t. the lexicographic ordering on the monomials in the $z_{i}^{ \pm 1}$. Therefore the images of the $\left(x^{(\sigma, \tau)}\right)^{l}\left(l \in \mathbb{Z}_{+}\right)$in $\mathcal{A}(\mathbb{T})$ are linearly independent, which completes the proof.

For every $l \in \mathbb{Z}_{+}$, any non-zero element of $\mathcal{H}^{(\sigma, \tau)}\left(l\left(\varepsilon_{1}-\varepsilon_{n}\right)\right)$ can be expressed as a polynomial of degree $l$ in $x^{(\sigma, \tau)}$. In order to identify these polynomials, we study the action of the following Casimir operator (cf. $[\mathrm{RTF}],[\mathrm{N}])$ on $\mathcal{H}^{(\sigma, \tau)}$ :

$$
C:=\sum_{i j} q^{2(n-i)} L_{i j}^{+} S\left(L_{j i}^{-}\right) \in \mathcal{U}_{q}
$$

The element $C \in \mathcal{U}_{q}$ is central and it acts as a scalar on each subspace $W(\lambda) \in \mathcal{A}_{q}$ $\left(\lambda \in P^{+}\right)$. The corresponding eigenvalue is given by

$$
\chi_{\lambda}(C):=\sum_{k=1}^{n} q^{2\left(\lambda_{k}+n-k\right)} .
$$

Since $C$ is central, the left action of $C$ on $\mathcal{A}_{q}$ preserves the subalgebra $\mathcal{H}^{(\sigma, \tau)}$. Hence it acts as a scalar on each subspace $\mathcal{H}^{(\sigma, \tau)}\left(l\left(\varepsilon_{1}-\varepsilon_{n}\right)\right)\left(l \in \mathbb{Z}_{+}\right)$:

$$
\chi_{l}(C):=\chi_{\lambda}(C)=q^{2(l+n-1)}+q^{-2 l}+\frac{q^{2}-q^{2 n-2}}{1-q^{2}}, \quad \lambda=l\left(\varepsilon_{1}-\varepsilon_{n}\right) .
$$

It follows from Proposition 5.3 that there is a uniquely determined linear operator $D: \mathcal{H}_{\mid \mathbb{T}}^{(\sigma, \tau)} \rightarrow \mathcal{H}_{\mid \mathbb{T}}^{(\sigma, \tau)}$ (called the radial part of the Casimir operator $C$ ) such that on $\mathcal{H}^{(\sigma, \tau)}$ we have

$$
{ }_{\mid \mathbb{T}} \circ C=D \circ{ }_{\mid \mathbb{T}},
$$

where the symbol $C$ denotes the left action of the element $C \in \mathcal{U}_{q}$ on $\mathcal{H}^{(\sigma, \tau)} \subset \mathcal{A}_{q}$. Let us define a linear operator $T_{q, z}: \mathbb{C}\left[z^{ \pm 1}\right] \rightarrow \mathbb{C}\left[z^{ \pm 1}\right]$ (called $q$-shift operator) by putting $T_{q, z} f(z):=f(q z)$. Recall the notation $A(z ; q)$ from $(2.7)$. 
Theorem 5.4. Let $\sigma, \tau$ be finite. The radial part $D: \mathcal{H}_{\mid \mathbb{T}}^{(\sigma, \tau)} \rightarrow \mathcal{H}_{\mid \mathbb{T}}^{(\sigma, \tau)}$ of the Casimir operator $C$ is equal to the following second-order q-difference operator:

$$
D_{A W}:=A\left(z ; q^{2}\right)\left(T_{q^{2}, z}-\mathrm{id}\right)+A\left(z^{-1} ; q^{2}\right)\left(T_{q^{-2}, z}-\mathrm{id}\right)+\frac{1-q^{2 n}}{1-q^{2}} \cdot \mathrm{id},
$$

with parameters $a, b, c, d$ given by

$$
a=-q^{\sigma+\tau+1}, \quad b=-q^{-\sigma-\tau+1}, \quad c=q^{\sigma-\tau+1}, \quad d=q^{-\sigma+\tau+2(n-2)+1} .
$$

Proof. The proof of this theorem is rather long and will be deferred to section 6 . The case $n=2$ was essentially proved in [K3, Lemma 5.1].

Theorem 5.5. Let $\sigma, \tau$ be finite. The $(\sigma, \tau)$-spherical functions in $\mathcal{H}^{(\sigma, \tau)}\left(l\left(\varepsilon_{1}-\varepsilon_{n}\right)\right)$ $\left(l \in \mathbb{Z}_{+}\right)$are spanned by

$$
p_{l}\left(x^{(\sigma, \tau)} ;-q^{\sigma+\tau+1},-q^{-\sigma-\tau+1}, q^{\sigma-\tau+1}, q^{-\sigma+\tau+2(n-2)+1} \mid q^{2}\right),
$$

where $p_{l}$ is an Askey-Wilson polynomial.

Proof. Recall that Askey-Wilson polynomials are characterized as the polynomial solutions of the second-order $q$-difference equation (2.6). Let $\varphi^{l}$ be a non-zero element in $\mathcal{H}^{(\sigma, \tau)}\left(l\left(\varepsilon_{1}-\varepsilon_{n}\right)\right)$. Then $D \varphi_{\mid \mathbb{T}}^{l}=\chi_{l}(C) \varphi_{\mid \mathbb{T}}^{l}$ by definition of $D$. By comparing (5.9) and (5.7) with (2.6) one sees that $\varphi_{\mid \mathbb{T}}^{l}$ is a scalar multiple of the Askey-Wilson polynomial of degree $l$ in the variable $\frac{1}{2}\left(z+z^{-1}\right)$. The theorem now follows by Proposition 5.3.

For $n=2$ this result agrees with [K3, Theorem 5.2]. As a corollary of Theorem 5.5 we have:

Proposition 5.6. Let $\sigma, \tau$ be finite. Let $d m_{q}(z)=d m_{a, b, c, d ; q}(z)$ denote the measure defined in the right-hand side of (2.4) and normalized in such a way that $\int d m_{q}(z)=1$ (cf. (2.5)). Then the Haar functional $h: \mathcal{A}_{q} \rightarrow \mathbb{C}$ is given on $\mathcal{H}^{(\sigma, \tau)}$ by

$$
h\left(P\left(x^{(\sigma, \tau)}\right)\right)=\int P\left(\frac{z+z^{-1}}{2}\right) d_{q^{2}} m(z) \quad(P \in \mathbb{C}[x]),
$$

where the parameters $a, b, c, d$ are defined as in Theorem 5.4.

Let us now suppose $\sigma$ finite, $\tau= \pm \infty$. As follows from Remark 3.12 and (3.10), the direct sum $\mathcal{H}^{(\sigma, \pm \infty)}(0) \oplus \mathcal{H}^{(\sigma, \pm \infty)}\left(\left(\varepsilon_{1}-\varepsilon_{n}\right)\right) \subset \mathcal{A}_{q}$ is spanned by the unit element $1 \in \mathcal{A}_{q}$ and

$$
x^{(\sigma, \infty)}:=q^{\sigma-1} \hat{y}_{n n}-q^{-2} \quad \text { resp. } \quad x^{(\sigma,-\infty)}:=q^{-\sigma-1} \hat{y}_{11}-1,
$$

where $\hat{y}_{11}, \hat{y}_{n n} \in \mathcal{C}_{q}^{\sigma}$ are given by (cf. Remark 3.12):

$$
\hat{y}_{i j}:=t_{i n}^{*} t_{j 1}+t_{i 1}^{*} t_{j n}+q^{\sigma+1} t_{i 1}^{*} t_{j 1}+q^{-1-\sigma} t_{i n}^{*} t_{j n} .
$$

Note that $\hat{y}_{i j}$ and $y_{i j}\left(q^{\sigma}=\sqrt{\frac{d}{c}}\right)$ differ by a scalar multiple.

Proposition 5.7. Let $\sigma$ be finite. The algebra $\mathcal{H}^{(\sigma, \pm \infty)}$ is polynomial in $x^{(\sigma, \pm \infty)}$ and hence commutative. 
Proof. Suppose $\tau=\infty$. We may view $x^{(\sigma, \infty)}$ as a left $\mathfrak{k}^{\sigma}$-invariant function in the algebra $\mathcal{B}_{q}^{\infty}$. Recall (cf. Proposition 3.11) that $\left(t_{n n}^{*} t_{n 1}\right)^{l} \in \mathcal{B}_{q}^{\infty}$ is a non-zero highest weight vector of weight $l\left(\varepsilon_{1}-\varepsilon_{n}\right)$ under the left action of $\mathcal{U}_{q}$. Since $\left(t_{n n}^{*} t_{n 1}\right)^{l}$ is (up to a non-zero scalar multiple) the highest weight component of $\left(x^{(\sigma, \infty)}\right)^{l}$, the component of $\left(x^{(\sigma, \infty)}\right)^{l}$ in $\mathcal{H}^{(\sigma, \infty)}\left(l\left(\varepsilon_{1}-\varepsilon_{n}\right)\right)$ is non-zero, whence the statement for $\tau=\infty$. The case $\tau=-\infty$ is handled in a similar way.

By applying the limit transition from Askey-Wilson polynomials to big $q$-Jacobi polynomials (cf. Proposition 2.2) we obtain

Theorem 5.8. Let us suppose that $\sigma$ is finite. The $(\sigma, \pm \infty)$-spherical functions in $\mathcal{H}^{(\sigma, \pm \infty)}\left(l\left(\varepsilon_{1}-\varepsilon_{n}\right)\right)\left(l \in \mathbb{Z}_{+}\right)$are spanned by

$$
P_{l}^{(n-2,0)}\left(x^{(\sigma, \infty)} ; q^{2 \sigma}, 1: q^{2}\right) \quad \text { resp. } \quad P_{l}^{(n-2,0)}\left(x^{(\sigma,-\infty)} ; q^{-2 \sigma+2(n-2)}, 1: q^{2}\right),
$$

where $P_{l}^{(n-2,0)}$ is a big q-Jacobi polynomial.

Proof. Let us for instance consider the case $\tau \rightarrow \infty$. Take $a:=q^{\tau}, c:=q^{2 \sigma}, d:=1$, $\alpha:=n-2, \beta:=0$. Then $2 a(c d)^{\frac{1}{2}} x^{(\sigma, \tau)} / q \rightarrow x^{(\sigma, \infty)}$ when $\tau \rightarrow \infty$. Moreover, the parameter values in Proposition 2.2 agree with those in Theorem 5.5, taking into account the fact that the Askey-Wilson polynomials $r_{l}(x ; a, b, c, d \mid q)$ are symmetric in the parameters $a, b, c, d$ up to a scalar multiple.

For $n=2$ Theorem 5.8 agrees with [NM1, Theorem 3] and [K3, Theorem 6.2].

Now let $\sigma= \pm \infty, \tau= \pm \sigma$. Then the direct sum $\mathcal{H}^{(\sigma, \pm \sigma)}(0) \oplus \mathcal{H}^{(\sigma, \pm \sigma)}\left(\left(\varepsilon_{1}-\varepsilon_{n}\right)\right) \subset$ $\mathcal{A}_{q}$ is spanned by the unit element $1 \in \mathcal{A}_{q}$ and

$$
\begin{aligned}
& x^{(\infty, \infty)}:=q^{-2}-q^{-2} t_{n n}^{*} t_{n n}, \quad x^{(-\infty,-\infty)}:=1-t_{11}^{*} t_{11}, \\
& x^{(\infty,-\infty)}:=q^{-2(n-1)} t_{1 n}^{*} t_{1 n}, \quad x^{(-\infty, \infty)}:=t_{n 1}^{*} t_{n 1}
\end{aligned}
$$

respectively.

Proposition 5.9. Let $\sigma= \pm \infty$. The algebra $\mathcal{H}^{(\sigma, \pm \sigma)}$ is polynomial in $x^{(\sigma, \pm \sigma)}$.

Proof. This can be proved using Theorem 3.9 and the description of the $*$-operation in terms of quantum principal minors (cf. (4.10), (4.11)). In fact, an easy argument by comparing degrees of monomials shows that the $\left(x^{(\sigma, \pm \sigma)}\right)^{m}(1 \leq m \leq l)$ are linearly independent. On the other hand, they are clearly contained in the subspace $\bigoplus_{1 \leq m \leq l} \mathcal{H}^{(\sigma, \pm \sigma)}\left(m\left(\varepsilon_{1}-\varepsilon_{n}\right)\right)$, which has dimension $l$ by Proposition 5.1. This proves the assertion.

Theorem 5.10. Let $\sigma= \pm \infty$. The $(\sigma, \pm \sigma)$-spherical functions in the spaces $\mathcal{H}^{(\sigma, \pm \sigma)}\left(l\left(\varepsilon_{1}-\varepsilon_{n}\right)\right)\left(l \in \mathbb{Z}_{+}\right)$are spanned by

$$
p_{l}^{(n-2,0)}\left(x^{(\sigma, \sigma)}: q^{2}\right) \quad \text { resp. } \quad p_{l}^{(0, n-2)}\left(x^{(\sigma,-\sigma)}: q^{2}\right),
$$

where $p_{l}^{(n-2,0)}$ and $p_{l}^{(0, n-2)}$ are little q-Jacobi polynomials.

Proof. We apply Proposition 2.3 to Theorem 5.5. Suppose for instance $\sigma=\tau$. In the case $\sigma \rightarrow \infty$ we take $a:=q^{\sigma}, \alpha:=0, \beta:=n-2$. In the case $\sigma \rightarrow-\infty$ we take $a:=q^{-\sigma}, \alpha:=0, \beta:=n-2$. As is easily checked, in both cases $2 a^{2} x^{(\sigma, \tau)} / q \rightarrow$ $-x^{( \pm \infty, \pm \infty)}$ when $\sigma \rightarrow \pm \infty$. Also, the parameter values in Proposition 2.3 agree with those in Theorem 5.5. The result now follows.

The result for the case $(\infty, \infty)$ is in agreement with [NYM, Theorem 4.7]. For $n=2$ see also [K3, Theorem 6.4]. 
Remark 5.11. It is easy to deduce from Theorem 5.8 that the restriction of the Casimir operator to the subalgebra $\mathcal{H}^{(\sigma, \pm \infty)}$ ( $\sigma$ finite) is essentially equal to the second-order $q$-difference operator diagonalized by the big $q$-Jacobi polynomials (cf. section 2). A similar statement holds for little $q$-Jacobi polynomials.

\section{Computation of the Radial part}

Throughout this section we assume that $\sigma, \tau$ are finite. We start by establishing some notation and recalling a few facts.

From the fact that multiplication $\mathcal{A}_{q} \otimes \mathcal{A}_{q} \rightarrow \mathcal{A}_{q}$ is a $\mathcal{U}_{q}$-bimodule homomorphism one easily deduces that

$$
\langle u, v \cdot a \cdot w\rangle=\langle w u v, a\rangle \quad\left(u, v, w \in \mathcal{U}_{q}, a \in \mathcal{A}_{q}\right) .
$$

Hence, an element $a \in \mathcal{A}_{q}$ is $(\sigma, \tau)$-biinvariant if and only if $\left\langle\mathcal{U}_{q} \mathfrak{k}^{\sigma}+\mathfrak{k}^{\tau} \mathcal{U}_{q}, a\right\rangle=0$. For $u, v \in \mathcal{U}_{q}$ we write $u \sim v$ if $u-v \in \mathcal{U}_{q} \mathfrak{k}^{\sigma}+\mathfrak{k}^{\tau} \mathcal{U}_{q}$.

Recall that the duality between $\mathcal{U}_{q}$ and $\mathcal{A}_{q}$ allows us to identify $\mathcal{A}_{q}$ with a vector subspace of the linear dual $\mathcal{U}_{q}^{*}$. Restricting to $\mathcal{H}^{(\sigma, \tau)}$ one has the following natural commutative diagram:

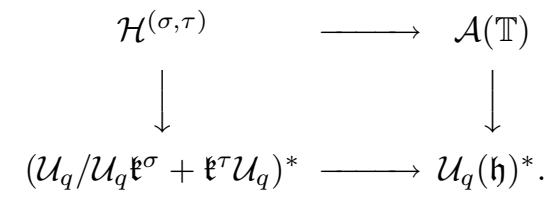

Here the vertical mappings and the upper horizontal mapping are injective. The goal of this section is to prove the following theorem:

Theorem 6.1. Let $C$ denote the Casimir operator defined in (5.5). If we put $\lambda:=\left\langle h, \varepsilon_{1}-\varepsilon_{n}\right\rangle\left(h \in P^{*}\right)$, then for generic $h \in P^{*}$ :

$$
q^{h} C \sim A\left(q^{\lambda} ; q^{2}\right)\left(q^{h+2 \varepsilon_{1}}-q^{h}\right)+A\left(q^{-\lambda} ; q^{2}\right)\left(q^{h-2 \varepsilon_{1}}-q^{h}\right)+\frac{1-q^{2 n}}{1-q^{2}} \cdot q^{h}
$$

with $A(z ; q)$ as defined in (2.7) and $a, b, c, d$ given by

$$
a=-q^{\sigma+\tau+1}, \quad b=-q^{-\sigma-\tau+1}, \quad c=q^{\sigma-\tau+1}, \quad d=q^{-\sigma+\tau+2(n-2)+1} .
$$

Here "generic" means that the denominators of $A\left(q^{\lambda} ; q^{2}\right)$ and $A\left(q^{-\lambda} ; q^{2}\right)$ are nonzero.

Proof. Let us first show that Theorem 6.1 implies Theorem 5.4. Write $x:=x^{(\sigma, \tau)} \in$ $\mathcal{H}^{(\sigma, \tau)}$ and recall (cf. Lemma 5.2) that $x_{\mid \mathbb{T}}=\frac{1}{2}\left(z+z^{-1}\right)$. Let $P_{l}$ be the unique monic polynomial (of degree $l$ ) such that $P_{l}(x) \in \mathcal{H}^{(\sigma, \tau)}\left(l\left(\varepsilon_{1}-\varepsilon_{n}\right)\right.$ ). Write $R_{l}(z):=$ $P_{l}\left(\frac{1}{2}\left(z+z^{-1}\right)\right)$ for the corresponding Laurent polynomial in $z$. Denote the $q$ difference operator defined in the right-hand side of (5.9) by $D_{A W}$. By definition of the radial part (cf. (5.8)) and by (6.2) it suffices to prove that for generic $h \in P^{*}$

$$
\left\langle q^{h}, C \cdot P_{l}(x)\right\rangle=\left\langle q^{h}, D_{A W} \cdot R_{l}(z)\right\rangle \quad\left(h \in P^{*}\right) .
$$

On the one hand we have

$$
\begin{aligned}
D_{A W} \cdot R_{l}(z) & =A\left(z ; q^{2}\right)\left(R_{l}\left(q^{2} z\right)-R_{l}(z)\right) \\
& -A\left(z^{-1} ; q^{2}\right)\left(R_{l}\left(q^{-2} z\right)-R_{l}(z)\right)+\frac{1-q^{2 n}}{1-q^{2}} R_{l}(z) .
\end{aligned}
$$


On the other hand, using (6.1) and (6.3) one gets

$$
\begin{aligned}
\left\langle q^{h}, C \cdot P_{l}(x)\right\rangle= & \left\langle q^{h} C, P_{l}(x)\right\rangle=\left\langle A\left(q^{\lambda} ; q^{2}\right)\left(q^{h+2 \varepsilon_{1}}-q^{h}\right)\right. \\
& \left.-A\left(q^{-\lambda} ; q^{2}\right)\left(q^{h-2 \varepsilon_{1}}-q^{h}\right)+\frac{1-q^{2 n}}{1-q^{2}} q^{h}, R_{l}(z)\right\rangle .
\end{aligned}
$$

Now recall that $\left\langle q^{h}, z\right\rangle=q^{\left\langle h, \varepsilon_{1}-\varepsilon_{n}\right\rangle}=: q^{\lambda}$ (cf. Proposition 5.3) and $\left\langle q^{\varepsilon_{1}}, z\right\rangle=q$. A comparison of (6.5) and (6.6) then yields (6.4).

The proof of Theorem 6.1 is based on the commutation relations (1.5) between the elements $L_{i j}^{ \pm} \in \mathcal{U}_{q}$. From (1.7) one derives that $L^{\varepsilon} S\left(L^{\varepsilon}\right)=S\left(L^{\varepsilon}\right) L^{\varepsilon}=I(\varepsilon= \pm$, $I$ the identity matrix). Using this identity one can rewrite (1.5) as

$$
S\left(L_{2}^{\varepsilon_{2}}\right) R^{+} L_{1}^{\varepsilon_{1}}=L_{1}^{\varepsilon_{1}} R^{+} S\left(L_{2}^{\varepsilon_{2}}\right)
$$

where $\left(\varepsilon_{1}, \varepsilon_{2}\right)$ is any of the three pairs $(+,+),(-,-),(+,-)$. Working this out explicitly, we get

Lemma 6.2. Suppose $1 \leq i, j, k, l \leq n$ and $\left(\varepsilon_{1}, \varepsilon_{2}\right) \in\{(+,+),(-,-),(+,-)\}$. Then one has the following identity:

$$
\begin{aligned}
q^{\delta_{i l}} S\left(L_{j l}^{\varepsilon_{2}}\right) L_{i k}^{\varepsilon_{1}} & +\left(q-q^{-1}\right) \delta_{i l} \sum_{m>i} S\left(L_{j m}^{\varepsilon_{2}}\right) L_{m k}^{\varepsilon_{1}} \\
& =q^{\delta_{k j}} L_{i k}^{\varepsilon_{1}} S\left(L_{j l}^{\varepsilon_{2}}\right)+\left(q-q^{-1}\right) \delta_{j k} \sum_{m<j} L_{i m}^{\varepsilon_{1}} S\left(L_{m l}^{\varepsilon_{2}}\right) .
\end{aligned}
$$

Let us introduce the following notation:

$$
\begin{aligned}
& C_{1}:=\sum_{k=2}^{n-1} q^{2(n-1)} L_{1 k}^{+} S\left(L_{k 1}^{-}\right), \quad C_{2}:=\sum_{k=2}^{n-1} q^{2(n-k)} L_{k n}^{+} S\left(L_{n k}^{-}\right), \\
& C_{3}:=q^{2(n-1)} L_{1 n}^{+} S\left(L_{n 1}^{-}\right), \quad C_{4}:=\sum_{i=1}^{n} q^{2(n-i)} L_{i i}^{+} S\left(L_{i i}^{-}\right) .
\end{aligned}
$$

Lemma 6.3. One has

$$
q^{h} C \sim q^{h} C_{1}+q^{h} C_{2}+q^{h} C_{3}+q^{h} C_{4} .
$$

Proof. This is immediate from the definition of $C$ (cf. (5.5)) and the form of the coideal $\mathfrak{k}^{\sigma}$ (cf. (3.1)).

The next step is to reduce each of the elements $q^{h} C_{i}(1 \leq i \leq 4)$ modulo $\mathcal{U}_{q} \mathfrak{k}^{\sigma}+\mathfrak{k}^{\tau} \mathcal{U}_{q}$ to a suitable element in $\mathcal{U}_{q}(\mathfrak{h})$. We shall do this in a series of lemmas in which we repeatedly apply the identities (6.8) and (1.6). It will be left to the reader to fill in most of the computational details, but with the indications we give this should be completely straightforward.

Let us introduce the following terminology. Let $X$ be a monomial in the $L_{k l}^{ \pm}$and suppose $L_{i j}^{ \pm}(1 \leq i, j \leq n$ fixed) occurs in (3.1) (r) $(\mathrm{r}=i, i i, \ldots, v i)$. Then it is clear that by using (3.1) (r) we can eliminate $L_{i j}^{ \pm}$in the monomial $L_{i j}^{ \pm} X$ modulo $\mathfrak{k}^{\tau} \mathcal{U}_{q}$. We call this procedure reduction on the left by (3.1) (r). Reduction on the right is defined in a similar way.

Observe (cf. (6.8)) that modulo $\mathcal{U}_{q} \mathfrak{k}^{\sigma}$ we have $(2 \leq k \leq n-1)$

$$
S\left(L_{k 1}^{-}\right) L_{1 k}^{+} \sim L_{1 k}^{+} S\left(L_{k 1}^{-}\right)+\left(1-q^{-2}\right) \sum_{m<k} L_{1 m}^{+} S\left(L_{m 1}^{-}\right)-\left(1-q^{-2}\right) .
$$

Using this we can prove 
Lemma 6.4. We have $(2 \leq k \leq n-1)$

$q^{h} L_{1 k}^{+} S\left(L_{k 1}^{-}\right) \sim-\left(1-q^{-2}\right)\left\{\sum_{1<m<k} q^{h} L_{1 m}^{+} S\left(L_{m 1}^{-}\right)+\frac{1+q^{\sigma+\tau+1+\lambda}}{1-q^{2 \lambda+2}}\left(q^{h+2 \varepsilon_{1}}-q^{h}\right)\right\}$.

Proof. We first reduce on the left by (3.1) (ii), apply (6.8), and then reduce on the right by (3.1) (ii). The result is:

$$
q^{h} L_{1 k}^{+} S\left(L_{k 1}^{-}\right) \sim q^{-\sigma+\tau-1-\lambda} q^{h} S\left(L_{k 1}^{-}\right) L_{1 k}^{+}+\left(1-q^{-2}\right) q^{\tau-\lambda} \sum_{m<k} q^{h} L_{n m}^{-} S\left(L_{m 1}^{-}\right) .
$$

Next, one eliminates $L_{n m}^{-}(1<m<k)$ and $L_{n 1}^{-}$by reduction on the left by (3.1) (ii) and (vi) respectively. Then apply (6.11) to get rid of the term $S\left(L_{k 1}^{-}\right) L_{1 k}^{+}$:

$$
\begin{aligned}
& q^{h} L_{1 k}^{+} S\left(L_{k 1}^{-}\right) \\
& \quad \sim q^{-\sigma+\tau-1-\lambda} q^{h} L_{1 k}^{+} S\left(L_{k 1}^{-}\right)+\left(1-q^{-2}\right)\left(q^{-\sigma+\tau-1-\lambda}-1\right) \sum_{1<m<k} q^{h} L_{1 m}^{+} S\left(L_{m 1}^{-}\right) \\
& \quad+\left(1-q^{-2}\right)\left(q^{-\sigma+\tau-1-\lambda}-\left(1-q^{2 \tau}\right)\right)\left(q^{h+2 \varepsilon_{1}}-q^{h}\right)+q^{\tau+\lambda}\left(1-q^{-2}\right) q^{h} L_{1 n}^{+} L_{11}^{+} .
\end{aligned}
$$

Reducing $q^{h} L_{1 n}^{+} L_{11}^{+}$first on the left, then on the right, by (3.1) (vi), one gets

$$
\left(1-q^{-2 \lambda-2}\right) q^{h} L_{1 n}^{+} L_{11}^{+} \sim\left(q^{-\lambda}\left(q^{-\tau}-q^{\tau}\right)-q^{-2 \lambda-1}\left(q^{-\sigma}-q^{\sigma}\right)\right)\left(q^{h+2 \varepsilon_{1}}-q^{h}\right) .
$$

Substituting the last formula into the preceding one, we arrive at the desired result.

Lemma 6.5. We have $(2 \leq k \leq n-1)$

$$
q^{h} L_{1 k}^{+} S\left(L_{k 1}^{-}\right) \sim-q^{-2(k-2)} \frac{\left(1-q^{-2}\right)\left(1+q^{\sigma+\tau+1+\lambda}\right)}{1-q^{2 \lambda+2}}\left(q^{h+2 \varepsilon_{1}}-q^{h}\right) .
$$

Proof. This follows from Lemma 6.4 by induction on $k$.

In other words, we can now write $q^{h} C_{1}$ as an element of $\mathcal{U}_{q}(\mathfrak{h})$ modulo $\mathcal{U}_{q} \mathfrak{k}^{\sigma}+\mathfrak{k}^{\tau} \mathcal{U}_{q}$, more precisely as a linear combination of $q^{h+2 \varepsilon_{1}}$ and $q^{h}$. We now deal with $q^{h} C_{2}$. Observe (cf. (6.8)) that modulo $\mathfrak{k}^{\tau} \mathcal{U}_{q}$ we have

$$
S\left(L_{n k}^{-}\right) L_{k n}^{+}+\left(1-q^{-2}\right) \sum_{m>k} S\left(L_{n m}^{-}\right) L_{m n}^{+} \sim L_{k n}^{+} S\left(L_{n k}^{-}\right)+\left(1-q^{-2}\right) .
$$

Lemma 6.6. We have $(2 \leq k \leq n-1)$

$$
q^{h} L_{k n}^{+} S\left(L_{n k}^{-}\right) \sim \frac{\left(q-q^{-1}\right) q^{-2 \lambda+1}\left(1+q^{-\sigma-\tau-1+\lambda}\right)}{1-q^{-2 \lambda+2}}\left(q^{h}-q^{h-2 \varepsilon_{1}}\right)
$$

Proof. First reduce on the left by (3.1) (iii), and then apply (6.8). In the resulting expression, $L_{k 1}^{-}$can be eliminated by reduction on the right using (3.1) (iii). One then obtains

$$
q^{h} L_{k n}^{+} S\left(L_{n k}^{-}\right) \sim q^{\sigma-\tau+1-\lambda} q^{h} S\left(L_{n k}^{-}\right) L_{k n}^{+}-q^{-\tau-\lambda}\left(q-q^{-1}\right) \sum_{m>k} q^{h} S\left(L_{n m}^{-}\right) L_{m 1}^{-} .
$$


Now apply (6.14) to get rid of $S\left(L_{n k}^{-}\right) L_{k n}^{+}$, eliminate $L_{m 1}^{-}(k<m<n)$ by reducing on the right by (3.1) (iii), and replace $L_{n n}^{ \pm}$by $L_{11}^{\mp}$ (cf. (3.1) (i)):

$$
\begin{aligned}
q^{h} L_{k n}^{+} S\left(L_{n k}^{-}\right) & \sim q^{\sigma-\tau+1-\lambda} q^{h} L_{k n}^{+} S\left(L_{n k}^{-}\right) \\
& +q^{\sigma-\tau-\lambda}\left(q-q^{-1}\right)\left(q^{h}-q^{h-2 \varepsilon_{1}}\right)-q^{-\tau-\lambda}\left(q-q^{-1}\right) q^{h} L_{11}^{-} L_{n 1}^{-} .
\end{aligned}
$$

Reducing $q^{h} L_{11}^{-} L_{n 1}^{-}$first on the right, then on the left by (3.1) (vi), one gets

$$
\left(1-q^{-2 \lambda+2}\right) q^{h} L_{11}^{-} L_{n 1}^{-} \sim\left(q^{-\lambda+1}\left(q^{-\tau}-q^{\tau}\right)+\left(q^{\sigma}-q^{-\sigma}\right)\right)\left(q^{h}-q^{h-2 \varepsilon_{1}}\right) .
$$

Substitution of the last formula into the preceding one yields the lemma.

Lemma 6.6 allows us to reduce $q^{h} C_{2}$ to a linear combination of $q^{h}$ and $q^{h-2 \varepsilon_{1}}$ modulo $\mathcal{U}_{q} \mathfrak{k}^{\sigma}+\mathfrak{k}^{\tau} \mathcal{U}_{q}$. We proceed with some preliminary results that will be used to reduce $q^{h} C_{3}$.

Lemma 6.7. We have

$$
\sum_{m>1} S\left(L_{n m}^{-}\right) L_{m 1}^{-}=\sum_{m>1} q^{-2(m-1)+1} L_{m 1}^{-} S\left(L_{n m}^{-}\right) .
$$

Proof. We prove the following more general statement $(1 \leq p \leq n)$ :

$$
\sum_{m>1} S\left(L_{n m}^{-}\right) L_{m 1}^{-}=\sum_{m=2}^{p} q^{-2(m-1)+1} L_{m 1}^{-} S\left(L_{n m}^{-}\right)+q^{-2(p-1)} \sum_{m>p} S\left(L_{n m}^{-}\right) L_{m 1}^{-} .
$$

The lemma follows from this by taking $p=n$. We prove (6.18) by induction on $p$. The assertion is trivial for $p=1$. Suppose it is true for a fixed $p(1 \leq p<n)$. We use the following identity $(1 \leq p<n)$, which follows in a straightforward way from (6.8):

$$
\sum_{m>p} S\left(L_{n m}^{-}\right) L_{m 1}^{-}=q^{-1} L_{p+1,1}^{-} S\left(L_{n, p+1}^{-}\right)+q^{-2} \sum_{m>p+1} S\left(L_{n m}^{-}\right) L_{m 1}^{-} .
$$

One now proves (6.18) with $p$ replaced by $p+1$ by first using the induction hypothesis and then applying (6.19).

Lemma 6.8. We have the following two equivalences:

$$
\begin{gathered}
q^{h} L_{11}^{+} S\left(L_{n 1}^{-}\right) \sim q^{-\tau+\lambda} \sum_{m=2}^{n-1} q^{h} L_{1 m}^{+} S\left(L_{m 1}^{-}\right) \\
\quad-q^{2 \lambda} q^{h} L_{1 n}^{+} L_{11}^{+}+q^{\lambda}\left(q^{-\tau}-q^{\tau}\right)\left(q^{h+2 \varepsilon_{1}}-q^{h}\right) \\
q^{h} L_{11}^{-} S\left(L_{n 1}^{-}\right) \sim q^{\tau+\lambda} \sum_{m=2}^{n-1} q^{-2(m-1)} q^{h} L_{m n}^{+} S\left(L_{n m}^{-}\right)-q^{-2(n-1)+1} q^{h} L_{11}^{-} L_{n 1}^{-}
\end{gathered}
$$

Proof. We start with the first equivalence. Reducing $q^{h} L_{11}^{+} S\left(L_{n 1}^{-}\right)$on the left by (3.1) (i) and using the fact that $\sum_{m=1}^{n} L_{n m}^{-} S\left(L_{m 1}^{-}\right)=0$, we get

$$
q^{h} L_{11}^{+} S\left(L_{n 1}^{-}\right) \sim-\sum_{m=2}^{n-1} q^{h} L_{n m}^{-} S\left(L_{m 1}^{-}\right)-q^{h} L_{n 1}^{-} S\left(L_{11}^{-}\right) .
$$


Next, we eliminate $L_{n m}^{-}(1<m<n)$ and $L_{n 1}^{-}$by reduction on the left using (3.1) (ii) and (vi) respectively, and simplify to obtain (6.20). To prove the second equivalence, observe that by the identity $\sum_{m=1}^{n} S\left(L_{n m}^{-}\right) L_{m 1}^{-}=0$ one has

$$
q^{h} L_{11}^{-} S\left(L_{n 1}^{-}\right) \sim-q^{-1} \sum_{m>1} q^{h} S\left(L_{n m}^{-}\right) L_{m 1}^{-} .
$$

Apply Lemma 6.7 to the right-hand side, reduce on the left by (3.1) (iii), and use reduction on the right by (3.1) (i) to eliminate $S\left(L_{n n}^{-}\right)=L_{n n}^{+}$. One then obtains (6.21).

Lemma 6.9. We have the following equivalence

$$
\begin{aligned}
& \left(1-q^{-2 \lambda}\right) q^{h} L_{1 n}^{+} S\left(L_{n 1}^{-}\right) \\
& \quad \sim\left(1-q^{-2}\right)\left\{q^{-2 \lambda} \sum_{m=2}^{n-1} q^{h} L_{1 m}^{+} S\left(L_{m 1}^{-}\right)-q^{-\sigma+\tau-1-\lambda} \sum_{m=2}^{n-1} q^{-2(m-2)} q^{h} L_{m n}^{+} S\left(L_{n m}^{-}\right)\right\} \\
& \quad+\left(1-q^{-2}\right) q^{-\sigma-2 \lambda}\left(q^{-2(n-2)}-1\right) q^{h} L_{11}^{-} L_{n 1}^{-} \\
& \quad+q^{-\lambda}\left(q^{-\tau}-q^{\tau}\right) q^{h}\left(L_{11}^{+}-L_{11}^{-}\right) S\left(L_{n 1}^{-}\right)-q^{-2 \lambda}\left(q^{-\sigma}-q^{\sigma}\right) q^{h} S\left(L_{n 1}^{-}\right)\left(L_{11}^{+}-L_{11}^{-}\right) \\
& \quad+\left(1-q^{-2}\right) q^{-2 \lambda}\left(q^{h+2 \varepsilon_{1}}-q^{h-2 \varepsilon_{1}}\right) .
\end{aligned}
$$

Proof. To prove this lemma, we start by reducing $q^{h} L_{1 n}^{+} S\left(L_{n 1}^{-}\right)$on the left by (3.1) (vi). This leads to

$$
q^{h} L_{1 n}^{+} S\left(L_{n 1}^{-}\right) \sim q^{-2 \lambda} q^{h} L_{n 1}^{-} S\left(L_{n 1}^{-}\right)+q^{-\lambda}\left(q^{-\tau}-q^{\tau}\right) q^{h}\left(L_{11}^{+}-L_{11}^{-}\right) S\left(L_{n 1}^{-}\right) .
$$

Now observe that by (6.8) one has $L_{n 1}^{-} S\left(L_{n 1}^{-}\right)=S\left(L_{n 1}^{-}\right) L_{n 1}^{-}$and

$$
\begin{aligned}
q S\left(L_{n 1}^{-}\right) L_{1 n}^{+}+ & \left(q-q^{-1}\right) \sum_{m>1} S\left(L_{n m}^{-}\right) L_{m n}^{+} \\
& =q L_{1 n}^{+} S\left(L_{n 1}^{-}\right)+\left(q-q^{-1}\right) \sum_{m<n} L_{1 m}^{+} S\left(L_{m 1}^{-}\right) .
\end{aligned}
$$

Reducing $q^{h} S\left(L_{n 1}^{-}\right) L_{n 1}^{-}$on the right by (3.1) (vi) and eliminating $S\left(L_{n 1}^{-}\right) L_{1 n}^{+}$by (6.23), one gets

$$
\begin{aligned}
q^{h} L_{n 1}^{-} & S\left(L_{n 1}^{-}\right) \sim q^{h} L_{1 n}^{+} S\left(L_{n 1}^{-}\right) \\
& +\left(1-q^{-2}\right) \sum_{m<n} q^{h} L_{1 m}^{+} S\left(L_{m 1}^{-}\right)-\left(1-q^{-2}\right) \sum_{m=2}^{n-1} q^{h} S\left(L_{n m}^{-}\right) L_{m n}^{+} \\
& -\left(q^{-\sigma}-q^{\sigma}\right) q^{h} S\left(L_{n 1}^{-}\right)\left(L_{11}^{+}-L_{11}^{-}\right)-\left(1-q^{-2}\right) q^{h-2 \varepsilon_{1}} .
\end{aligned}
$$

Consider the term $\sum_{m=2}^{n-1} q^{h} S\left(L_{n m}^{-}\right) L_{m n}^{+}$in (6.24). We reduce it on the right by (3.1) (iii), apply Lemma 6.7, and then eliminate $L_{m 1}^{-}(1<m<n)$ and $L_{n 1}^{-}$by reducing on the left by (3.1) (iii) and (vi) respectively. One ends up with

$$
\begin{aligned}
& \sum_{m=2}^{n-1} q^{h} S\left(L_{n m}^{-}\right) L_{m n}^{+} \\
& \quad \sim q^{-\sigma+\tau-1+\lambda} \sum_{m=2}^{n-1} q^{-2(m-2)} q^{h} L_{m n}^{+} S\left(L_{n m}^{-}\right)+q^{-\sigma}\left(1-q^{2(n-2)}\right) q^{h} L_{11}^{-} L_{n 1}^{-}
\end{aligned}
$$

Now substitute the last formula into (6.24), and the resulting equation into (6.22). One then obtains the lemma. 
Lemma 6.10. We have

$$
q^{h} C_{4} \sim q^{2(n-1)} q^{h+2 \varepsilon_{1}}+q^{h-2 \varepsilon_{1}}+\frac{q^{2}-q^{2(n-1)}}{1-q^{2}} q^{h} .
$$

Proof. This follows by reduction using (3.1) (i) and (v).

We conclude from Lemma 6.3 and the subsequent lemmas that $q^{h} C$ can be written modulo $\mathcal{U}_{q} \mathfrak{k}^{\sigma}+\mathfrak{k}^{\tau} \mathcal{U}_{q}$ as a linear combination of $q^{h+2 \varepsilon_{1}}, q^{h}$, and $q^{h-2 \varepsilon_{1}}$. To conclude the proof of Theorem 6.1 we have to compute explicit expressions for the coefficients in this linear combination.

We start with the coefficient of $q^{h+2 \varepsilon_{1}}$, which we denote by $\left(q^{h} C\right)^{+}$. Collecting terms and using the identity

$$
\sum_{k=2}^{n-1} q^{-2(k-2)}=\frac{1-q^{-2(n-2)}}{1-q^{-2}}
$$

we get the following expression for $\left(q^{h} C\right)^{+}$:

$$
\begin{aligned}
& -\frac{\left(q^{2(n-1)}-q^{2}\right)\left(1+q^{\sigma+\tau+1+\lambda}\right)}{1-q^{2 \lambda+2}}-\frac{\left(1-q^{-2}\right)\left(q^{2(n-1)}-q^{2}\right) q^{-2 \lambda}\left(1+q^{\sigma+\tau+1+\lambda}\right)}{\left(1-q^{-2 \lambda}\right)\left(1-q^{2 \lambda+2}\right)} \\
& -\frac{\left(q^{2(n-1)}-q^{2}\right)\left(1+q^{\sigma+\tau+1+\lambda}\right) q^{-\tau+\lambda} M}{\left(1-q^{-2 \lambda}\right)\left(1-q^{2 \lambda+2}\right)}-\frac{q^{2(n-1)} q^{2 \lambda} M^{2}}{\left(1-q^{-2 \lambda}\right)\left(1-q^{-2 \lambda-2}\right)} \\
& +\frac{q^{2(n-1)} q^{\lambda}\left(q^{-\tau}-q^{\tau}\right) M}{1-q^{-2 \lambda}}+\frac{q^{2(n-1)}\left(1-q^{-2}\right) q^{-2 \lambda}}{1-q^{-2 \lambda}}+q^{2(n-1)},
\end{aligned}
$$

where $M:=q^{-\lambda}\left(q^{-\tau}-q^{\tau}\right)-q^{-2 \lambda-1}\left(q^{-\sigma}-q^{\sigma}\right)$. Clearly, the expression in (6.26) can be uniquely written as a quotient of a polynomial $N\left(q^{\lambda}\right)$ in the variable $q^{\lambda}$ of degree four and the polynomial $\left(1-q^{2 \lambda}\right)\left(1-q^{2 \lambda+2}\right)$. A completely elementary but rather tedious calculation shows that the coefficients of $N\left(q^{\lambda}\right)=: \sum_{i=0}^{4} n_{i} q^{i \lambda}$ are given by

$$
\begin{aligned}
& n_{0}=1, \quad n_{1}=q^{\sigma+\tau+1}+q^{-\sigma-\tau+1}-q^{\sigma-\tau+1}-q^{-\sigma+\tau+2(n-2)+1}, \\
& n_{2}=q^{2(n-2)+2}-q^{-2 \sigma+2(n-2)+2}-q^{-2 \tau+2}-q^{2 \tau+2(n-2)+2}-q^{2 \sigma+2}+q^{2}, \\
& n_{3}=q^{\sigma+\tau+2(n-2)+3}+q^{-\sigma-\tau+2(n-2)+3}-q^{\sigma-\tau+3}-q^{-\sigma+\tau+2(n-2)+3}, \\
& n_{4}=q^{2 n} .
\end{aligned}
$$

From this it easily follows that $\left(q^{h} C\right)^{+}$is equal to

$$
\frac{\left(1+q^{\sigma+\tau+1+\lambda}\right)\left(1+q^{-\sigma-\tau+1+\lambda}\right)\left(1-q^{\sigma-\tau+1+\lambda}\right)\left(1-q^{-\sigma+\tau+2(n-2)+1+\lambda}\right)}{\left(1-q^{2 \lambda}\right)\left(1-q^{2 \lambda+2}\right)},
$$

in other words $\left(q^{h} C\right)^{+}=A\left(q^{\lambda} ; q^{2}\right)$ with $a, b, c, d$ as given in Theorem 6.1. In a similar way, one can prove that the coefficients of $q^{h-2 \varepsilon_{1}}$ and $q^{h}$ in the reduction of $q^{h} C$ are as given in Theorem 6.1. We shall leave the details to the reader. However, there is a shortcut argument to prove Theorem 5.4 from here. Reasoning as we did to deduce Theorem 5.4 from Theorem 6.1, one sees that the results proved so far imply that the radial part $D$ is a linear combination with rational coefficients in $z$ of $T_{q^{2}, z}, T_{q^{-2}, z}$ and id. The coefficient of $T_{q^{2}, z}$ is equal to $A\left(z ; q^{2}\right)$ with parameters as given in Theorem 5.4. Now let $w: \mathbb{C}\left[z^{ \pm 1}\right] \rightarrow \mathbb{C}\left[z^{ \pm 1}\right]$ be the unique algebra automorphism sending $z$ to $z^{-1}$. The radial part $D$ commutes with $w$, since its 
eigenfunctions are $w$-invariant by Proposition 5.3. On the other hand, one has the relation $w \circ T_{q, z}=T_{q^{-1}, z} \circ w$. This implies that the coefficient of $T_{q^{-2}, z}$ in the expression for $D$ is equal to $A\left(z^{-1} ; q^{2}\right)$. Hence, the radial part $D$ and the operator $D_{A W}$ defined in (5.9) differ at most a multiple of the identity. To show that they are actually equal, it clearly suffices to exhibit a non-zero $P \in \mathbb{C}\left[z+z^{-1}\right]$ such that $D \cdot P=D_{A W} \cdot P$. Let us take $P=1$. Then, on the one hand, we have

$$
D \cdot 1=C \cdot 1=\varepsilon(C) 1=\sum_{i=1}^{n} q^{2(n-i)} 1=\frac{1-q^{2 n}}{1-q^{2}} 1,
$$

whereas, on the other hand, $D_{A W} \cdot 1=\frac{1-q^{2 n}}{1-q^{2}} 1$ by (5.9). This completes the proof of Theorem 5.4.

\section{REFERENCES}

[AA1] G.E. Andrews, R. Askey, Enumeration of partitions: the role of Eulerian series and q-orthogonal polynomials, Higher Combinatorics (M. Aigner, ed.), Reidel, Boston, MA, 1977, pp. 3-26. MR 80b:10021

[AA2] Classical orthogonal polynomials, Polynômes Orthogonaux et Applications (C. Brezinski, A. Draux, A.P. Magnus, P. Maroni, A. Ronveaux, eds.), Lecture Notes in Math. 1171, Springer-Verlag, Berlin, 1985, pp. 36-62. MR 88c:33015b

[AW] R. Askey, J. Wilson, Some basic hypergeometric orthogonal polynomials that generalize Jacobi polynomials, Mem. Amer. Math. Soc. 54 (319) (1985), 1-55. MR 87a:05023

[Dz] M.S. Dijkhuizen, Some remarks on the construction of quantum symmetric spaces, Acta Appl. Math. 44 (1996), 59-80. CMP 96:17

[DK1] M.S. Dijkhuizen, T.H. Koornwinder, Quantum homogeneous spaces, duality and quantum 2-spheres, Geom. Dedicata 52 (1994), 291-315. MR 95i:16037

[DK2] , CQG algebras: a direct algebraic approach to compact quantum groups, Lett. Math. Phys. 32 (1994), 315-330. MR 95m:16029

[Dr1] V.G. Drinfel'd, Quantum groups, Proceedings ICM Berkeley (1986) (A.M. Gleason, ed.), Amer. Math. Soc., Providence, RI, 1986, pp. 798-820. MR 89f:17017

[Dr2] _ On almost cocommutative Hopf algebras, Leningrad Math. J. 1 (2) (1990), 321342. MR 91b:16046

[GR] G. Gasper, M. Rahman, Basic hypergeometric series, Encyclopedia of Mathematics and Its Applications 35, Cambridge University Press, London, 1990. MR 91d:33034

[J] M. Jimbo, A q-analogue of $U(\mathfrak{g l}(n))$, Hecke algebra and the Yang-Baxter equation, Lett. Math. Phys. 11 (1986), 247-252. MR 87k:17011

[Kk] H.T. Koelink, The addition formula for continuous $q$-Legendre polynomials and associated spherical elements on the $S U(2)$ quantum group related to Askey-Wilson polynomials, SIAM J. Math. Anal. 25 (1) (1994), 197-217. MR 95f:33023

[K1] T.H. Koornwinder, Representations of the twisted SU(2) quantum group and some $q$ hypergeometric orthogonal polynomials, Indag. Math. 51 (1989), 97-117. MR 90h:17016

[K2] Orthogonal polynomials in connection with quantum groups, Orthogonal polynomials: Theory and Practice (P. Nevai, ed.), NATO-ASI Series C 294, Kluwer, Norwell, MA, 1990, pp. 257-292. MR 91i:33018

[K3] Askey-Wilson polynomials as zonal spherical functions on the SU(2) quantum group, SIAM J. Math. Anal. 24 (3) (1993), 795-813. MR 94k:33042

[KV] L.I. Korogodsky, L.L. Vaksman, Quantum G-spaces and Heisenberg algebra, Quantum Groups (P.P. Kulish, ed.), Lecture Notes in Math. 1510, Springer-Verlag, Berlin, 1992, pp. 56-66. MR 93k:17031

[L] G. Lusztig, Quantum deformations of certain simple modules over enveloping algebras, Adv. Math. 70 (1988), 237-249. MR 89k:17029

[M] T. Masuda, K. Mimachi, Y. Nakagami, M. Noumi, K. Ueno, Representations of quantum groups and a q-analogue of orthogonal polynomials, C. R. Acad. Sci. Paris Sér. I Math. 307 (1988), 559-564. MR 90a:17013

[N] M. Noumi, Macdonald's symmetric polynomials as zonal spherical functions on some quantum homogeneous spaces, Adv. Math. 123 (1996), 16-77. MR 98a:33004 
[NM1] M. Noumi, K. Mimachi, Quantum 2-spheres and big q-Jacobi polynomials, Comm. Math. Phys. 128 (1990), 521-531. MR 91f:33010

[NM2] _ Askey-Wilson polynomials and the quantum group $S U_{q}(2)$, Proc. Japan Acad. Ser. A Math. Sci. 66 (1990), 146-149. MR 91k:33015

[NM3] S Spherical functions on a family of quantum 3-spheres, Compositio Math. 83 (1992), 19-42. MR 93f:33010

[NS1] M. Noumi, T. Sugitani, Quantum symmetric spaces and related $q$-orthogonal polynomials, Group Theoretical Methods in Physics (A. Arima et al., eds.), Proceedings XX ICGTMP, Toyonaka (Japan), 1994, World Scientific, Singapore, 1995, pp. 28-40. MR 97h:33033

[NS2] , Quantum symmetric spaces and multivariable orthogonal polynomials, preprint (1996).

[NYM] M. Noumi, H. Yamada, K. Mimachi, Finite-dimensional representations of the quantum group $G L_{q}(n, \mathbb{C})$ and the zonal spherical functions on $U_{q}(n-1) \backslash U_{q}(n)$, Japanese J. Math. 19 (1) (1993), 31-80. MR 94i:17023

[P] P. Podleś, Quantum spheres, Lett. Math. Phys. 14 (1987), 193-202. MR 89b:46081

[RTF] N. Reshetikhin, L.A. Takhtadzhyan, L.D. Faddeev, Quantization of Lie groups and Lie algebras, Leningrad Math. J. 1 (1990), 193-225. MR 90j:17039

[R] M. Rosso, Finite-dimensional representations of the quantum analog of a complex simple Lie algebra, Comm. Math. Phys. 117 (1988), 581-593. MR 90c:17019

[So] Ya. S. Solbel'man, Irreducible representations of the function algebra on the quantum group $S U(n)$, and Schubert cells, Soviet Math. Dokl. 40 (1) (1990), 34-38. MR 91d:17019

[SV] Ya. S. Soubel'man, L.L. Vaksman, On some problems in the theory of quantum groups, Representation Theory and Dynamical Systems (A.M. Vershik, ed.), Adv. Sov. Math., vol. 9, Amer. Math. Soc., Providence, RI, 1992, pp. 3-55. MR 93h:17043

[S1] T. Sugitani, Harmonic analysis on quantum spheres associated with representations of $\mathcal{U}_{q}\left(\mathfrak{s o}_{N}\right)$ and $q$-Jacobi polynomials, Compositio Math. 99 (1995), 249-281. MR 96k:33029

[S2] Zonal spherical functions on quantum Grassmann manifolds, preprint (1996).

[VS1] L.L. Vaksman, Y.S. Solbel'man, Algebra of functions on the quantum group SU(2), Funct. Anal. Appl. 22 (1988), 170-181. MR 90f:17019

[VS2] The algebra of functions on the quantum group $S U(n+1)$ and odd-dimensional quantum spheres, Leningrad Math. J. 2 (5), 1023-1042. MR 92e:58021

[W] S.L. Woronowicz, Compact matrix pseudogroups, Comm. Math. Phys. 111 (1987), 613665. MR 88m:46079

Department of Mathematics, Faculty of Science, Kobe University, Rokko, Kobe 657, JAPAN

E-mail address: msdz@math.s.kobe-u.ac.jp

Department of Mathematics, Faculty of Science, Kobe University, Rokko, Kobe 657, JAPAN

E-mail address: noumi@math.s.kobe-u.ac.jp 